JOURNAL OF

FUNCTION SPACES AND APPLICATIONS

Volume 5, Number 1 (2007), 49-88
(C) 2007, Scientific Horizon

http://www.jfsa.net

\title{
A note on maximal operator on $\ell^{\left\{p_{n}\right\}}$ and $L^{p(x)}(\mathbb{R})$
}

\author{
Aleš Nekvinda* \\ (Communicated by Pankaj Jain)
}

2000 Mathematics Subject Classification. 46E30, 26D15.

Keywords and phrases. Generalized Lebesque space, maximal operator.

\begin{abstract}
We consider a discrete analogue of Hardy-Littlewood maximal operator on the generalized Lebesque space $\ell^{\left\{p_{n}\right\}}$ of sequences defined on $\mathbb{Z}$. It is known a necessary and sufficient condition $\mathcal{P}$ which guarantees an existence of a real number $p>1$ such that the norms in the space $\ell^{\left\{p_{n}\right\}}$ and in the classical space $\ell^{p}$ are equivalent. Of course, this condition immediately implies the boundedness of maximal operator on $\ell^{\left\{p_{n}\right\}}$ and, moreover, $\lim _{|n| \rightarrow \infty} p_{n}=p$. We construct two examples of sequences $\left\{p_{n}\right\}$ satisfying $\lim _{|n| \rightarrow \infty} p_{n}=p$ in this paper. In the first example the maximal operator is unbounded on $\ell^{\left\{p_{n}\right\}}$ and the sequence $\left\{p_{n}\right\}$ from the second example does not satisfy $\mathcal{P}$ but the maximal operator is bounded. Moreover, it is known a sufficient integral condition to a behavior of a function $p(x)$ at infinity which guarantees the boundedness of the maximal operator on $L^{p(.)}\left(\mathbb{R}^{n}\right)$. As a main result of this paper we construct a function $p(x)$ which does not satisfy this integral condition nevertheless the maximal operator is bounded.
\end{abstract}

${ }^{*}$ The author thanks especially to Dr. Lars Diening for personal comunication and many contributions. The author was supported by MSM 6840770010. 


\section{Introduction}

The generalized Lebesque spaces $L^{p(x)}(\Omega)$ and its discrete analogy $\ell^{\left\{p_{n}\right\}}$ are investigated more and more in recent years. We refer to [17] for the establishment of the fundamental properties of these spaces, to [7] for some properties of the norm of $L^{p(x)}(\Omega)$ and to [22,23] for some motivation where non-linear system of partial differential equation with coefficients of variable rate of growth are studied.

In [8] and [20] a discrete version $\ell^{\left\{p_{n}\right\}}$ of $L^{p(x)}$ is introduced and some interesting properties of these spaces are proved. Especially, a necessary and sufficient condition to bounded sequences $\left\{p_{n}\right\}$ and $\left\{q_{n}\right\}$ is found to guarantee the equivalency of norms in spaces $\ell^{\left\{p_{n}\right\}}$ and $\ell^{\left\{q_{n}\right\}}$. In $[9,10]$ the imbedding $W^{1, p(x)}(\Omega) \hookrightarrow L^{q(x)}(\Omega)$, an analogy of Sobolev imbedding $W^{1, p}(\Omega) \hookrightarrow L^{q}(\Omega)$, is proved. In [11] a sufficient condition for compactness of the imbedding $W^{1, p(x)}(\Omega) \hookrightarrow L^{q(x)}(\Omega)$ is found.

For further information on properties of $L^{p(x)}$ spaces and singular operators spaces see $[3,4,5,6,13,14,15,16]$.

In [2] L.Diening proved that the maximal operator is bounded on $L^{p(x)}(\Omega)$ for a bounded $\Omega$ provided $-|p(x)-p(y)| \ln |x-y| \leq C$ for $|x-y| \leq \frac{1}{2}$, $x, y \in \Omega$. In [19] the authors showed that the maximal operator can be unbounded if there exists $x \in \Omega$ such that $\lim _{y \rightarrow x}|p(x)-p(y)| \ln |x-y|=$ $-\infty$.

The partial generalization of this problem to the domain $\mathbb{R}^{n}$ is done in [1] where a sufficient condition for boundedness of maximal operator on $L^{p(x)}\left(\mathbb{R}^{n}\right)$ is found for a certain class of functions $p(x)$. A more general sufficient condition is found in [21] for a wider class of functions $p(x)$.

As a natural motivation for the investigation of the behavior of maximal operator on $L^{p(x)}(\mathbb{R})$ can serve a discrete version of maximal operator on $\ell^{\left\{p_{n}\right\}}$. We will consider in the next a set of all real sequences $\left\{a_{n}\right\}_{n \in \mathbb{Z}}:=$ $\left\{a_{n}\right\}$ defined on $\mathbb{Z}$. Given a real number $p \geq 1$ denote by $\mathcal{B}_{p}$ the set of all "exponent sequences" $\left\{p_{n}\right\}, 1 \leq p_{n}$ and $\lim _{|n| \rightarrow \infty} p_{n}=p$.

It is known (see [21]) a necessary and sufficient condition $\mathcal{P}$ for sequence $\left\{p_{n}\right\}$ to guarantee an existence of a real number $r \geq 1$ such that norms in $\ell^{\left\{p_{n}\right\}}$ and in $\ell^{r}$ are equivalent. Of course, if a sequence $\left\{p_{n}\right\}$ satisfies $\mathcal{P}$ then by classical results on maximal operator on $\ell^{r}$ we immediately obtain the boundedness of maximal operator on $\ell^{\left\{p_{n}\right\}}$.

On the other hand we construct in this paper in Section 5 an example of $\left\{p_{n}\right\} \in \mathcal{B}_{r} \backslash \mathcal{P}$ such that the maximal operator is unbounded on $\ell^{\left\{p_{n}\right\}}$.

In connection with this example the natural problem appears. Does there exist a sequence $\left\{p_{n}\right\} \in \mathcal{B}_{r} \backslash \mathcal{P}$ such that the maximal operator is bounded on $\ell^{\left\{p_{n}\right\}}$ ? In Section 5 an answer to this question is given and it is positive. 
We construct a $\left\{p_{n}\right\} \in \mathcal{B}_{r} \backslash \mathcal{P}$ such that the maximal operator is bounded on $\ell^{\left\{p_{n}\right\}}$.

In [8] an example of a Lipschitz function $p(x)$ defined on $\mathbb{R}$ is given such that $M$ is unbounded on $L^{p(x)}(\mathbb{R})$. But $p$ has no limit at infinity.

In [21] there is found a sufficient condition $\mathcal{P}$ to the behavior of a function $p$ at infinity to guarantee the boundedness of $M$ on $L^{p(x)}(\mathbb{R})$. The main result of this paper is contained in Section 6 where we construct a function $p(x)$ which does not satisfy the condition $\mathcal{P}$ but $M$ is bounded on $L^{p(x)}(\mathbb{R})$.

\section{Preliminaries}

In this section the basic notations, definitions and lemmas are given. We

consider in this paper mappings defined on integers. Given $\mathfrak{a}: \mathbb{Z} \rightarrow \mathbb{R}$ we speak about a sequence $\mathfrak{a}=\left\{a_{n}\right\}_{n \in \mathbb{Z}}$.

Let $p \geq 1$. Denote

$$
\begin{aligned}
& \mathcal{M}=\left\{\mathfrak{a} ; a_{n} \in \mathbb{R}\right\}, \quad \mathcal{M}_{+}=\left\{\mathfrak{a} ; a_{n} \geq 0\right\} \\
& \mathcal{B}=\left\{\mathfrak{p} \in \mathcal{M} ; \text { there is } K \geq 1 \text { such that } 1 \leq p_{n} \leq K\right\}, \\
& \mathcal{B}_{p}=\left\{\mathfrak{p} \in \mathcal{B} ; \lim _{|n| \rightarrow \infty} p_{n}=p\right\} .
\end{aligned}
$$

Definition 2.1. Let $\mathfrak{p} \in \mathcal{B}$. Denote for $\mathfrak{a} \in \mathcal{M}$ the Luxenburg norm by

$$
\|\mathfrak{a}\|_{\mathfrak{p}}=\inf \left\{\lambda>0 ; \sum_{n \in \mathbb{Z}}\left|\frac{a_{n}}{\lambda}\right|^{p_{n}} \leq 1\right\} .
$$

Define a space $\ell^{\mathfrak{p}}$ by

$$
\ell^{\mathfrak{p}}=\left\{\mathfrak{a} \in \mathcal{M} ;\|\mathfrak{a}\|_{\mathfrak{p}}<\infty\right\} .
$$

Recall that for a constant sequence $\mathfrak{p}$ such that $p_{n}=p$ we obtain the well-known classical space $\ell^{p}$ equipped with the norm

$$
\|\mathfrak{a}\|=\left(\sum_{n \in \mathbb{Z}}\left|a_{n}\right|^{p}\right)^{1 / p}
$$

In [8] (see Lemma 2.6) the following lemma is proved.

Lemma 2.2. Let $\mathfrak{p} \in \mathcal{B}$. Then

$$
\ell^{\mathfrak{p}}=\left\{\mathfrak{a} \in \mathcal{M} ; \sum_{n \in \mathbb{Z}}\left|a_{n}\right|^{p_{n}}<\infty\right\} .
$$


In what follows we will use the idea of one-sides maximal operators which was used for instance in [18]. Let us define a discrete version of maximal operator and its one-sides modifications.

Definition 2.3. Given $\mathfrak{a} \in \mathcal{M}$ we define operators $M^{-}, M^{+}$and $M$ by

$$
\begin{aligned}
\left(M^{-} \mathfrak{a}\right)_{n} & =\sup _{k \leq n} \frac{1}{n-k+1} \sum_{j=k}^{n}\left|a_{j}\right|, \\
\left(M^{+} \mathfrak{a}\right)_{n} & =\sup _{n \leq k} \frac{1}{k-n+1} \sum_{j=n}^{k}\left|a_{j}\right| \\
(M \mathfrak{a})_{n} & =\sup _{k \leq n \leq l} \frac{1}{l-k+1} \sum_{j=k}^{l}\left|a_{j}\right| .
\end{aligned}
$$

Lemma 2.4. The inequality

$$
(M \mathfrak{a})_{j} \leq 2\left(\left(M^{+} \mathfrak{a}\right)_{j}+\left(M^{-} \mathfrak{a}\right)_{j}\right)
$$

holds for each $j \in \mathbb{Z}$ and $a \in \mathcal{M}$.

Proof. Let $a \in \mathcal{M}$ and fix $j \in \mathbb{Z}$. Find integers $m, n \geq 0$ such that

$$
(M \mathfrak{a})_{j} \leq \frac{2}{m+n+1} \sum_{s=j-m}^{j+n}\left|a_{s}\right| .
$$

Now, we can write

$$
\begin{aligned}
(M \mathfrak{a})_{j} & \leq \frac{2}{m+n+1} \sum_{s=j-m}^{j+n}\left|a_{s}\right| \\
& =\frac{2(m+1)}{m+n+1} \frac{1}{m+1} \sum_{s=j-m}^{j}\left|a_{s}\right|+\frac{2(n+1)}{m+n+1} \frac{1}{n+1} \sum_{s=j}^{j+n}\left|a_{s}\right| \\
& \leq 2\left(\frac{1}{m+1} \sum_{s=j-m}^{j}\left|a_{s}\right|+\frac{1}{n+1} \sum_{s=j}^{j+n}\left|a_{s}\right|\right) \\
& \leq 2\left(\left(M^{-} \mathfrak{a}\right)_{j}+\left(M^{+} \mathfrak{a}\right)_{j}\right)
\end{aligned}
$$

which proves the lemma.

Remind that the Hardy-Littlewood maximal operator is defined by

$$
\mathfrak{M} f(x)=\sup _{a<x<b} \frac{1}{b-a} \int_{a}^{b}|f(x)| d x
$$


for all $f \in L_{l o c}^{1}(\mathbb{R})$. Define one-side maximal operators by

$$
\mathfrak{M}^{+} f(x)=\sup _{x<b} \frac{1}{b-x} \int_{x}^{b}|f(x)| d x, \quad \mathfrak{M}^{-} f(x)=\sup _{a<x} \frac{1}{x-a} \int_{a}^{x}|f(x)| d x .
$$

Recall a classical theorem (see [12], Theorem 21.76 (i)).

Lemma 2.5. Let $p>1$. Then the inequality

$$
\left(\int_{\mathbb{R}}\left(\mathfrak{M}^{+} f(x)\right)^{p} d x\right)^{1 / p} \leq \frac{p}{p-1}\left(\int_{\mathbb{R}}|f(x)|^{p} d x\right)^{1 / p}
$$

holds for all $f \in L^{p}(\mathbb{R})$. The same inequality holds for $\mathfrak{M}^{-}$.

Lemma 2.6. Let $p>1$. Then the inequality

$$
\left(\int_{\mathbb{R}}(\mathfrak{M} f(x))^{p} d x\right)^{1 / p} \leq \frac{2 p}{p-1}\left(\int_{\mathbb{R}}|f(x)|^{p} d x\right)^{1 / p}
$$

holds for all $f \in L^{p}(\mathbb{R})$.

Proof. Clearly,

$$
\begin{aligned}
\mathfrak{M} f(x) & =\sup _{a<x<b} \frac{1}{b-a} \int_{a}^{b}|f(x)| d x \\
& =\sup _{a<x<b} \frac{1}{b-a}\left(\int_{a}^{x}|f(x)| d x+\int_{x}^{b}|f(x)| d x\right) \\
& \leq \sup _{a<x<b} \frac{1}{b-a} \int_{a}^{x}|f(x)| d x+\sup _{a<x<b} \frac{1}{b-a} \int_{x}^{b}|f(x)| d x \\
& \leq \sup _{a<x} \frac{1}{x-a} \int_{a}^{x}|f(x)| d x+\sup _{x<b} \frac{1}{b-x} \int_{x}^{b}|f(x)| d x \\
& =\mathfrak{M}^{+} f(x)+\mathfrak{M}^{-} f(x) .
\end{aligned}
$$

Thus, we have by Lemma 2.5

$$
\begin{aligned}
\left(\int_{\mathbb{R}}(\mathfrak{M} f(x))^{p} d x\right)^{1 / p} & \leq\left(\int_{\mathbb{R}}\left(\mathfrak{M}^{+} f(x)\right)^{p} d x\right)^{1 / p}+\left(\int_{\mathbb{R}}\left(\mathfrak{M}^{-} f(x)\right)^{p} d x\right)^{1 / p} \\
& \leq \frac{2 p}{p-1}\left(\int_{\mathbb{R}}|f(x)|^{p} d x\right)^{1 / p}
\end{aligned}
$$

which finishes the proof.

In what follows we will use the discrete analogue of the previous lemma for modular inequality. 
Lemma 2.7. Let $1<p<q<\infty$. Then there exists $C_{p, q}>0$ such that for each real number $r, p \leq r \leq q$, and for any $\mathfrak{a} \in \mathcal{M}_{+}$the inequality

$$
\sum_{j \in \mathbb{Z}}(M \mathfrak{a})_{j}^{r} \leq C_{p, q} \sum_{j \in \mathbb{Z}} a_{j}^{r}
$$

holds.

Proof. Consider first the operator $M^{+}$. Let $\mathfrak{a} \in \mathcal{M}_{+}$. Set $f(x)=$ $\sum_{k \in \mathbb{Z}} a_{k} \chi_{(k, k+1)}(x)$. Fix $k \in \mathbb{Z}$ and $x \in(k, k+1)$. Then for any $m \geq 0$

$$
\begin{aligned}
\frac{1}{m+1} \sum_{j=k}^{k+m} a_{j} & =\frac{1}{m+1} \sum_{j=k}^{k+m} \int_{j}^{j+1} a_{j} \chi_{(j, j+1)}(x) d x \\
& =\frac{1}{m+1} \sum_{j=k}^{k+m} \int_{j}^{j+1} f(x) d x \\
& =\frac{1}{m+1} \int_{k}^{k+m+1} f(x) d x \\
& \leq \mathfrak{M} f(x) .
\end{aligned}
$$

Thus,

$$
\left(M^{+} \mathfrak{a}\right)_{k}=\sup _{m \geq 0} \frac{1}{m+1} \sum_{k}^{k+m} a_{j} \leq \mathfrak{M} f(x) \quad \text { for all } \quad x \in(k, k+1)
$$

and so,

$$
\left(M^{+} \mathfrak{a}\right)_{k}^{r} \leq \int_{k}^{k+1}(\mathfrak{M} f(x))^{r} d x .
$$

Consequently, we have by Lemma 2.6

$$
\begin{aligned}
\sum_{k \in \mathbb{Z}}\left(M^{+} \mathfrak{a}\right)_{k}^{r} & \leq \sum_{k \in \mathbb{Z}} \int_{k}^{k+1}(\mathfrak{M} f(x))^{r} d x \\
& =\int_{\mathbb{R}}(\mathfrak{M} f(x))^{r} d x \\
& \leq\left(\frac{2 r}{r-1}\right)^{r} \int_{\mathbb{R}}(f(x))^{r} d x \\
& =\left(\frac{2 r}{r-1}\right)^{r} \sum_{k \in \mathbb{Z}} \int_{k}^{k+1} a_{k}^{r} \chi_{(k, k+1)}(x) d x \\
& =\left(\frac{2 r}{r-1}\right)^{r} \sum_{k \in \mathbb{Z}} a_{k}^{r} .
\end{aligned}
$$


Analogously we can prove an estimate

$$
\sum_{k \in \mathbb{Z}}\left(M^{-} \mathfrak{a}\right)_{k}^{r} \leq\left(\frac{2 r}{r-1}\right)^{r} \sum_{k \in \mathbb{Z}} a_{k}^{r} .
$$

Using (2.1) we have

$$
\begin{aligned}
\sum_{k \in \mathbb{Z}}(M \mathfrak{a})_{k}^{r} & \leq 2^{2 r-1}\left(\sum_{k \in \mathbb{Z}}\left(M^{+} \mathfrak{a}\right)_{k}^{r}+\sum_{k \in \mathbb{Z}}\left(M^{-} \mathfrak{a}\right)_{k}^{r}\right) \\
& \leq 2^{2 r}\left(\frac{2 r}{r-1}\right)^{r} \sum_{k \in \mathbb{Z}} a_{k}^{r} .
\end{aligned}
$$

Setting

$$
C_{p, q}=\max _{p \leq r \leq q} 2^{2 r}\left(\frac{2 r}{r-1}\right)^{r}
$$

we obtain the desired inequality.

Definition 2.8. Let $\mathfrak{s} \in \mathcal{M}$. Say that $\mathfrak{s}$ satisfies a condition $\mathcal{P}$ (write $\mathfrak{s} \in \mathcal{P})$ if there exists a real number $c>0$ such that

$$
\sum_{\left\{n \in \mathbb{Z} ; s_{n}>0\right\}} s_{n} c^{1 / s_{n}}<\infty .
$$

Definition 2.9. Let $p>1$. Say that $\mathfrak{p} \in \mathcal{B}$ satisfies a condition $\mathcal{P}_{p}$ (write $\mathfrak{p} \in \mathcal{P}_{p}$ ) if $\mathfrak{p}-p:=\left\{p_{n}-p\right\}_{n \in \mathbb{Z}} \in \mathcal{P}$, i.e. there exists a real number $c>0$ such that

$$
\sum_{\left\{n \in \mathbb{Z} ;\left|p_{n}-p\right|>0\right\}}\left|p_{n}-p\right| c^{1 /\left|p_{n}-p\right|}<\infty .
$$

The following basic lemma is proved in [21] (see Theorem 4.3).

Lemma 2.10. Let $\mathfrak{p}, \mathfrak{q} \in \mathcal{B}$ be given sequences. Then $|\mathfrak{p}-\mathfrak{q}| \in \mathcal{P}$ if and only if the norms in $\ell^{\mathfrak{p}}$ and $\ell^{\mathfrak{q}}$ are equivalent. Especially, assuming $|\mathfrak{p}-\mathfrak{q}| \in \mathcal{P}$ there is a constant $C>0$ such that the implication

$$
\sum_{j \in \mathbb{Z}} b_{j}^{q_{j}} \leq 1 \Rightarrow \sum_{j \in \mathbb{Z}} b_{j}^{p_{j}} \leq C
$$

holds.

As an easy consequence we obtain the following two lemmas.

Lemma 2.11. Let $\mathfrak{p} \in \mathcal{B}$ and $p>1$. Then the norms in $\ell^{\mathfrak{p}}$ and $\ell^{p}$ are equivalent if and only if $\mathfrak{p} \in \mathcal{P}_{p}$. 
Lemma 2.12. Let $\mathfrak{p}, \mathfrak{q} \in \mathcal{B}$ be given sequences, $q_{n} \geq p_{n}$. Let $C$ be the constant from Lemma 2.10. Then

$$
\sum_{j \in \mathbb{Z}} b_{j}^{q_{j}} \leq B \Rightarrow \sum_{j \in \mathbb{Z}} b_{j}^{p_{j}} \leq \max \left\{1, \sup _{j \in \mathbb{Z}} B^{\frac{p_{j}}{q_{j}}}\right\} C .
$$

As an easy consequence of Lemma 2.7 and Lemma 2.11 we obtain the following lemma.

Lemma 2.13. Let $p>1, \mathfrak{p} \in \mathcal{P}_{p}$. Then $M$ is bounded on $\ell^{\mathfrak{p}}$.

Lemma 2.14. Let $p>1$. Then $\mathcal{P}_{p} \subset \mathcal{B}_{p}$.

Proof. Let

$$
\sum_{\left\{n \in \mathbb{Z} ;\left|p_{n}-p\right|>0\right\}}\left|p_{n}-p\right| c^{1 /\left|p_{n}-p\right|}<\infty
$$

for some positive $c$. Without lose of generality we can suppose $c \leq 1$. Assume that $\mathfrak{p} \notin \mathcal{B}_{p}$. Then there exists $\varepsilon>0$ and $\left\{n_{k}\right\}_{k=1}^{\infty}$ such that $\left|p_{n_{k}}-p\right|>\varepsilon$ for each $k$. Since $c \leq 1$ we have $c^{\frac{1}{p_{n_{k}}-p \mid}} \geq c^{\frac{1}{\varepsilon}}$ which implies

$$
\begin{aligned}
\infty & >\sum_{\left\{n \in \mathbb{Z} ;\left|p_{n}-p\right|>0\right\}}\left|p_{n}-p\right| c^{1 /\left|p_{n}-p\right|} \\
& \geq \sum_{k=1}^{\infty}\left|p_{n_{k}}-p\right| c^{1 /\left|p_{n_{k}}-p\right|} \\
& \geq \sum_{k=1}^{\infty} \varepsilon c^{\frac{1}{\varepsilon}} \\
& =\infty
\end{aligned}
$$

and gives a contradiction.

Definition 2.15. Say that the operator $M$ is bounded if there exists $C>0$ such that

$$
\sum_{n \in \mathbb{Z}} a_{n}^{p_{n}} \leq 1 \Rightarrow \sum_{n \in \mathbb{Z}}(M \mathfrak{a})_{n}^{p_{n}} \leq C
$$

holds for all $\mathfrak{a} \in \mathcal{M}_{+}$. Analogously we would define the boundedness of $\mathrm{M}^{+}$ and $M^{-}$.

In the next we will consider a special class of exponent sequences $\mathfrak{p}$. Given a non-decreasing sequence of positive integers $\left\{K_{n}\right\}_{n=1}^{\infty}$ we adopt a notation

$$
A_{0}=0, A_{n}=\sum_{j=1}^{n} K_{j}
$$


and define for $n \geq 1$ sets

$$
\mathcal{K}_{n}=\left\{j ; 1+A_{n-1} \leq j \leq A_{n}\right\} .
$$

Definition 2.16. Let $p>1$. Say that $\mathfrak{p} \in \mathcal{B}_{p}$ satisfies a condition $\mathcal{S}_{p}$ (write $p \in \mathcal{S}_{p}$ ) if there exists a non-decreasing sequence of positive integers $\left\{K_{n}\right\}_{n=1}^{\infty}$ and sequence of real numbers $\left\{r_{n}\right\}_{n=0}^{\infty}, r_{n} \searrow 0$, such that

$$
p_{j}= \begin{cases}p+r_{n} & \text { if } j \in \mathcal{K}_{n} \quad n=1,2, \ldots \\ p+r_{0} & \text { if } j=0 \\ p_{-j} & \text { if } j<0 .\end{cases}
$$

It is easy to see the following lemma.

Lemma 2.17. The inclusion $\mathcal{S}_{p} \subset \mathcal{B}_{p}$ holds.

\section{Unboundedness of $M$}

Proposition 3.1. Let $p>1$. Let $\mathfrak{p} \in \mathcal{S}_{p}$ and $\left\{K_{n}\right\}_{n=1}^{\infty},\left\{r_{n}\right\}_{n=1}^{\infty}$ be corresponding sequences. Assume that a sequence $\left\{K_{n}^{r_{n}-r_{n+1}}\right\}_{n=1}^{\infty}$ is unbounded. Then $M$ is unbounded on $\ell^{\mathfrak{p}}$.

Proof. Set $\varepsilon_{n}=1-\frac{p+r_{n+1}}{p+r_{n}}$. Since $K_{n}^{r_{n}-r_{n+1}}$ is unbounded then $K_{n}^{\varepsilon_{n}}$ is unbounded and consequently, there is a sequence $\left\{b_{n}\right\}_{n=1}^{\infty}$ such that

$$
\sum_{n=1}^{\infty} b_{n}^{1-\varepsilon_{n}} \leq 1 \text { and } \sum_{n=1}^{\infty} K_{n}^{\varepsilon_{n}} b_{n}^{1-\varepsilon_{n}}=\infty .
$$

Set $c_{n}=\left(\frac{b_{n}}{K_{n}}\right)^{\frac{1}{p+r_{n}}}$ and define $\mathfrak{a} \in \mathcal{M}$ by

$$
a_{j}= \begin{cases}c_{n} & \text { if } j \in \mathcal{K}_{n} \\ 0 & \text { if } j \leq 0\end{cases}
$$

Then

$$
\begin{aligned}
\sum_{j \in \mathbb{Z}} a_{j}^{p_{j}} & =\sum_{n=1}^{\infty} \sum_{j \in \mathcal{K}_{n}} a_{j}^{p+r_{n}} \\
& =\sum_{n=1}^{\infty} K_{n} c_{n}^{p+r_{n}}=\sum_{n=1}^{\infty} b_{n} \\
& \leq \sum_{n=1}^{\infty} b_{n}^{1-\varepsilon_{n}} \leq 1
\end{aligned}
$$


It is easy to see that we have for $j \in \mathcal{K}_{n+1}$

$$
\left(M^{-} \mathfrak{a}\right)_{j} \geq \frac{K_{n} c_{n}}{K_{n}+j-A_{n}}
$$

which implies

$$
\begin{aligned}
& \sum_{j \in \mathbb{Z}}(M \mathfrak{a})_{j}^{p_{j}} \geq \sum_{j \in \mathbb{Z}}\left(M^{-} \mathfrak{a}\right)_{j}^{p_{j}} \\
& \geq \sum_{n=1}^{\infty}\left(M^{-} \mathfrak{a}\right)_{j}^{p_{j}} \\
& =\sum_{n=1}^{\infty} \sum_{j \in \mathcal{K}_{n}}\left(M^{-} \mathfrak{a}\right)_{j}^{p_{j}} \\
& \geq \sum_{n=1}^{\infty}\left(K_{n} c_{n}\right)^{p+r_{n+1}} \sum_{j=1+A_{n}}^{A_{n+1}} \frac{1}{\left(K_{n}+j-A_{n}\right)^{p+r_{n+1}}} \\
& =\sum_{n=1}^{\infty}\left(K_{n} c_{n}\right)^{p+r_{n+1}} \sum_{s=1}^{K_{n+1}} \frac{1}{\left(K_{n}+s\right)^{p+r_{n+1}}} \\
& \geq \sum_{n=1}^{\infty}\left(K_{n} c_{n}\right)^{p+r_{n+1}} \sum_{s=1}^{K_{n}} \frac{1}{\left(K_{n}+s\right)^{p+r_{n+1}}} \\
& \geq \sum_{n=1}^{\infty}\left(K_{n} c_{n}\right)^{p+r_{n+1}} \sum_{s=1}^{K_{n}} \frac{1}{\left(2 K_{n}\right)^{p+r_{n+1}}} \\
& \geq \frac{1}{2^{p+r_{0}}} \sum_{n=1}^{\infty} K_{n} c_{n}^{p+r_{n+1}} \\
& =\frac{1}{2^{p+r_{0}}} \sum_{n=1}^{\infty} K_{n}\left(\frac{b_{n}}{K_{n}}\right)^{\frac{p+r_{n+1}}{p+r_{n}}} \\
& =\frac{1}{2^{p+r_{0}}} \sum_{n=1}^{\infty} K_{n}^{\varepsilon_{n}} b_{n}^{1-\varepsilon_{n}} \\
& =\infty \text {. }
\end{aligned}
$$

\section{Boundedness of $M$}

Lemma 4.1. Assume that $\mathfrak{p}$ given by (2.3) satisfies the following conditions: There exists a constant $D \geq 1$ such that 
(i) for a sequence $\mathfrak{q}$ given by

$$
q_{j}= \begin{cases}p+r_{n} & \text { if } j \in \mathcal{K}_{n+1} \quad n=0,1,2, \ldots \\ p+r_{0} & \text { if } j=0 \\ q_{-j} & \text { if } j<0\end{cases}
$$

we have $\mathfrak{q}-\mathfrak{p} \in \mathcal{P}$, i.e. there is $c>0$ such that

$$
\sum_{n=1}^{\infty}\left(r_{n}-r_{n+1}\right) c^{\frac{1}{r_{n}-r_{n+1}}} \leq D
$$

(ii) the inequality

$$
K_{m}^{1-\frac{1}{p+r_{m}}} \leq D K_{n}^{1-\frac{1}{p+r_{n}}}
$$

holds for all $n, m \in \mathbb{N}, m \leq n$.

(iii) the inequality

$$
K_{n}^{r_{n}-r_{n+1}} \leq D .
$$

holds for each $n \in \mathbb{N}$;

(iv) the inequality

$$
n K_{n}^{1-\frac{1}{p+r_{n+1}}} \leq D K_{n+1}^{1-\frac{1}{p+r_{n+1}}}
$$

holds for each $n \in \mathbb{N}$;

Then there exists $C>0$ such that the implication

$$
\sum_{j \in \mathbb{Z}} a_{j}^{p_{j}} \leq 1 \Rightarrow \sum_{j \in \mathbb{Z}}\left(M^{-} \mathfrak{a}\right)_{j}^{p_{j}} \leq C
$$

is satisfied for all $\mathfrak{a} \in \mathcal{M}_{+}$with $a_{n}=0$ for $n \leq 0$.

Proof. Let

$$
\sum_{j \in \mathbb{Z}} a_{j}^{p_{j}}=\sum_{j=1}^{\infty} a_{j}^{p_{j}}=\sum_{n=1}^{\infty} \sum_{j \in \mathcal{K}_{n}} a_{j}^{p+r_{n}} \leq 1 .
$$

Fix $n \in \mathbb{N}$ and $j \in \mathcal{K}_{n+1}$. Estimate $\left(M^{-} \mathfrak{a}\right)_{j}$. We can easily write

$$
\mathfrak{a}=\sum_{m=1}^{\infty} \mathfrak{a}^{(m)} \text { where } \mathfrak{a}^{(m)}=\mathfrak{a} \chi_{\mathcal{K}_{m}} .
$$


Since $M^{-}$is sub-linear we obtain

$$
\left(M^{-} \mathfrak{a}\right)_{j} \leq \sum_{m=1}^{\infty}\left(M^{-} \mathfrak{a}^{(m)}\right)_{j}
$$

Due to the fact $\left(M^{-} \mathfrak{a}^{(m)}\right)_{j}=0$ for $m>n+1$ we have for each $n \in \mathbb{N}$ (with a convention $\sum_{m=1}^{0}=0$ )

$$
\left(M^{-} \mathfrak{a}\right)_{j} \leq \sum_{m=1}^{n-1}\left(M^{-} \mathfrak{a}^{(m)}\right)_{j}+\left(M^{-} \mathfrak{a}^{(n)}\right)_{j}+\left(M^{-} \mathfrak{a}^{(n+1)}\right)_{j}
$$

This gives

$$
\begin{aligned}
& \sum_{j \in \mathbb{Z}}\left(M^{-} \mathfrak{a}\right)_{j}^{p_{j}} \\
& =\sum_{j=1}^{\infty}\left(M^{-} \mathfrak{a}\right)_{j}^{p_{j}} \\
& =\sum_{n=1}^{\infty} \sum_{j \in \mathcal{K}_{n}}\left(M^{-} \mathfrak{a}\right)_{j}^{p+r_{n}} \\
& =\sum_{j \in \mathcal{K}_{1}}\left(M^{-} \mathfrak{a}\right)_{j}^{p+r_{1}}+\sum_{n=2}^{\infty} \sum_{j \in \mathcal{K}_{n}}\left(M^{-} \mathfrak{a}\right)_{j}^{p+r_{n}} \\
& =\sum_{j \in \mathcal{K}_{1}}\left(M^{-} \mathfrak{a}\right)_{j}^{p+r_{1}}+\sum_{n=1}^{\infty} \sum_{j \in \mathcal{K}_{n+1}}\left(M^{-} \mathfrak{a}\right)_{j}^{p+r_{n+1}} \\
& \leq \sum_{j \in \mathcal{K}_{1}}\left(M^{-} \mathfrak{a}\right)_{j}^{p+r_{1}}+\sum_{n=1}^{\infty} \sum_{j \in \mathcal{K}_{n+1}}\left\{\sum_{m=1}^{n-1}\left(M^{-} \mathfrak{a}^{(m)}\right)_{j}+\left(M^{-} \mathfrak{a}^{(n)}\right)_{j}\right. \\
& \left.+\left(M^{-} \mathfrak{a}^{(n+1)}\right)_{j}\right\}^{p+r_{n+1}} \\
& \leq \sum_{j \in \mathcal{K}_{1}}\left(M^{-} \mathfrak{a}\right)_{j}^{p+r_{1}}+3^{p+r_{1}} \sum_{n=1}^{\infty}\left\{\sum_{j \in \mathcal{K}_{n+1}}\left(\sum_{m=1}^{n-1}\left(M^{-} \mathfrak{a}^{(m)}\right)_{j}\right)^{p+r_{n+1}}\right. \\
& \left.+\sum_{j \in \mathcal{K}_{n+1}}\left(M^{-} \mathfrak{a}^{(n)}\right)_{j}^{p+r_{n+1}}+\sum_{j \in \mathcal{K}_{n+1}}\left(M^{-} \mathfrak{a}^{(n+1)}\right)_{j}^{p+r_{n+1}}\right\} \\
& =\sum_{j \in \mathcal{K}_{1}}\left(M^{-} \mathfrak{a}\right)_{j}^{p+r_{1}}+3^{p+r_{1}} \sum_{n=1}^{\infty} \sum_{j \in \mathcal{K}_{n+1}}\left(\sum_{m=1}^{n-1}\left(M^{-} \mathfrak{a}^{(m)}\right)_{j}\right)^{p+r_{n+1}} \\
& +3^{p+r_{1}} \sum_{n=1}^{\infty} \sum_{j \in \mathcal{K}_{n+1}}\left(M^{-} \mathfrak{a}^{(n)}\right)_{j}^{p+r_{n+1}}+3^{p+r_{1}} \sum_{n=2}^{\infty} \sum_{j \in \mathcal{K}_{n}}\left(M^{-} \mathfrak{a}^{(n)}\right)_{j}^{p+r_{n}} .
\end{aligned}
$$


Since $\sum_{j \in \mathcal{K}_{1}}\left(M^{-} \mathfrak{a}\right)_{j}^{p+r_{1}}=\sum_{j \in \mathcal{K}_{1}}\left(M^{-} \mathfrak{a}^{(1)}\right)_{j}^{p+r_{1}}$ we obtain

$$
\begin{aligned}
\sum_{j \in \mathbb{Z}}\left(M^{-} \mathfrak{a}\right)_{j}^{p_{j}} \leq 3^{p+r_{1}} \sum_{n=1}^{\infty} \sum_{j \in \mathcal{K}_{n+1}}\left(\sum_{m=1}^{n-1}\left(M^{-} \mathfrak{a}^{(m)}\right)_{j}\right)^{p+r_{n+1}} \\
+3^{p+r_{1}} \sum_{n=1}^{\infty} \sum_{j \in \mathcal{K}_{n+1}}\left(M^{-} \mathfrak{a}^{(n)}\right)_{j}^{p+r_{n+1}} \\
+3^{p+r_{1}} \sum_{n=1}^{\infty} \sum_{j \in \mathcal{K}_{n}}\left(M^{-} \mathfrak{a}^{(n)}\right)_{j}^{p+r_{n}} \\
:=3^{p+r_{1}}\left(I_{1}+I_{2}+I_{3}\right) .
\end{aligned}
$$

Estimate first $I_{1}$. Using the convention $\sum_{m=1}^{0}=0$ we can write

$$
\begin{aligned}
I_{1} & =\sum_{n=1}^{\infty} \sum_{j \in \mathcal{K}_{n+1}}\left(\sum_{m=1}^{n-1}\left(M^{-} \mathfrak{a}^{(m)}\right)_{j}\right)^{p+r_{n+1}} \\
& =\sum_{n=2}^{\infty} \sum_{j \in \mathcal{K}_{n+1}}\left(\sum_{m=1}^{n-1}\left(M^{-} \mathfrak{a}^{(m)}\right)_{j}\right)^{p+r_{n+1}}
\end{aligned}
$$

and we have for $1 \leq m<n$ and $j \in \mathcal{K}_{n+1}$

$$
\begin{aligned}
\left(M^{-} \mathfrak{a}^{(m)}\right)_{j} & \leq \frac{1}{K_{m+1}+K_{m+2}+\cdots+K_{n}+j-A_{n}} \sum_{s \in \mathcal{K}_{m}} a_{s} \\
& \leq \frac{1}{K_{n}+j-A_{n}} \sum_{s \in \mathcal{K}_{m}} a_{s} .
\end{aligned}
$$

Moreover,

$$
\begin{aligned}
\sum_{j \in \mathcal{K}_{n+1}} \frac{1}{\left(K_{n}+j-A_{n}\right)^{p+r_{n+1}}} & =\sum_{s=1}^{K_{n+1}} \frac{1}{\left(K_{n}+s\right)^{p+r_{n+1}}} \\
& \leq \sum_{s=1}^{\infty}\left(K_{n}+s\right)^{-\left(p+r_{n+1}\right)} \\
& \leq \int_{K_{n}}^{\infty} x^{-\left(p+r_{n+1}\right)} d x \\
& =\frac{1}{p+r_{n+1}-1} K_{n}^{-p-r_{n+1}+1} \\
& \leq \frac{1}{p-1} K_{n}^{-p-r_{n+1}+1}
\end{aligned}
$$


and so,

$$
\begin{aligned}
I_{1} & =\sum_{n=2}^{\infty} \sum_{j \in \mathcal{K}_{n+1}}\left(\sum_{m=1}^{n-1}\left(M^{-} \mathfrak{a}^{(m)}\right)_{j}\right)^{p+r_{n+1}} \\
& \leq \sum_{n=2}^{\infty} \sum_{j \in \mathcal{K}_{n+1}}\left(\sum_{m=1}^{n-1}\left(\frac{1}{K_{n}+j-A_{n}} \sum_{s \in \mathcal{K}_{m}} a_{s}\right)\right)^{p+r_{n+1}} \\
& =\sum_{n=2}^{\infty} \sum_{j \in \mathcal{K}_{n+1}} \frac{1}{\left(K_{n}+j-A_{n}\right)^{p+r_{n+1}}}\left(\sum_{m=1}^{n-1} \sum_{s \in \mathcal{K}_{m}} a_{s}\right)^{p+r_{n+1}} \\
& \leq \frac{1}{p-1} \sum_{n=2}^{\infty} \frac{1}{K_{n}^{p+r_{n+1}-1}}\left(\sum_{m=1}^{n-1} \sum_{s \in \mathcal{K}_{m}} a_{s}\right)^{p+r_{n+1}} .
\end{aligned}
$$

Using the Hölder inequality we obtain

$$
\begin{aligned}
I_{1} & \leq \frac{1}{p-1} \sum_{n=2}^{\infty} \frac{1}{K_{n}^{p+r_{n+1}-1}}\left(\sum_{m=1}^{n-1}\left(\sum_{s \in \mathcal{K}_{m}} 1\right)^{\frac{p+r_{m}-1}{p+r_{m}}}\left(\sum_{s \in \mathcal{K}_{m}} a_{s}^{p+r_{m}}\right)^{\frac{1}{p+r_{m}}}\right)^{p+r_{n+1}} \\
& =\frac{1}{p-1} \sum_{n=2}^{\infty} \frac{1}{K_{n}^{p+r_{n+1}-1}}\left(\sum_{m=1}^{n-1} K_{m}^{\frac{p+r_{m}-1}{p+r_{m}}}\left(\sum_{s \in \mathcal{K}_{m}} a_{s}^{p+r_{m}}\right)^{\frac{1}{p+r_{m}}}\right)^{p+r_{n+1}}
\end{aligned}
$$

Using (4.1) we have $\sum_{s \in \mathcal{K}_{m}} a_{s}^{p+r_{m}} \leq \sum_{m=1}^{\infty} \sum_{s \in \mathcal{K}_{m}} a_{s}^{p+r_{m}} \leq 1$ which gives

$$
I_{1} \leq \frac{1}{p-1} \sum_{n=2}^{\infty} \frac{1}{K_{n}^{p+r_{n+1}-1}}\left(\sum_{m=1}^{n-1} K_{m}^{1-\frac{1}{p+r_{m}}}\right)^{p+r_{n+1}} .
$$

Now, if $n \geq 3$ we have by (ii) an inequality $K_{m}^{1-\frac{1}{p+r_{m}}} \leq D K_{n-2}^{1-\frac{1}{p+r_{n}-2}}$ for $1 \leq m \leq n-2$ which gives

$$
\begin{aligned}
J & :=\sum_{m=1}^{n-1} K_{m}^{1-\frac{1}{p+r_{m}}} \\
& =K_{n-1}^{1-\frac{1}{p+r_{n}-1}}+\sum_{m=1}^{n-2} K_{m}^{1-\frac{1}{p+r_{m}}} \\
& \leq K_{n-1}^{1-\frac{1}{p+r_{n-1}}}+D \sum_{m=1}^{n-2} K_{n-2}^{1-\frac{1}{p+r_{n-2}}} \\
& =K_{n-1}^{1-\frac{1}{p+r_{n-1}}}+D(n-2) K_{n-2}^{1-\frac{1}{p+r_{n-2}}} .
\end{aligned}
$$


By (iii) we have $K_{n-2}^{r_{n-2}-r_{n-1}} \leq D$ and so,

$$
K_{n-2}^{\frac{\left(p+r_{n-2}\right)-\left(p+r_{n-1}\right)}{\left(p+r_{n-1}\right)\left(p+r_{n-2}\right)}} \leq D^{\frac{1}{\left(p+r_{n}-1\right)\left(p+r_{n-2}\right)}} \leq D
$$

which gives

$$
K_{n-2}^{\frac{1}{p+r_{n-1}}-\frac{1}{p+r_{n-2}}} \leq D
$$

and finally,

$$
K_{n-2}^{1-\frac{1}{p+r_{n-2}}} \leq D K_{n-2}^{1-\frac{1}{p+r_{n-1}}}
$$

This shows with (4.5) and (iv)

$$
\begin{aligned}
J & \leq K_{n-1}^{1-\frac{1}{p+r_{n-1}}}+(n-2) D K_{n-2}^{1-\frac{1}{p+r_{n-1}}} \\
& \leq K_{n-1}^{1-\frac{1}{p+r_{n-1}}}+D^{2} K_{n-1}^{1-\frac{1}{p+r_{n-1}}} \\
& =\left(1+D^{2}\right) K_{n-1}^{1-\frac{1}{p+r_{n}-1}} .
\end{aligned}
$$

If $n=2$ then $\sum_{m=1}^{n-1} K_{m}^{1-\frac{1}{p+r_{m}}}=K_{1}^{1-\frac{1}{p+r_{1}}}=K_{n-1}^{1-\frac{1}{p+r_{n-1}}}$. In both cases we obtain

$$
\sum_{m=1}^{n-1} K_{m}^{1-\frac{1}{p+r_{m}}} \leq\left(1+D^{2}\right) K_{n-1}^{1-\frac{1}{p+r_{n-1}}}
$$

which gives with (4.4)

$$
\begin{aligned}
I_{1} & \leq \frac{1}{p-1} \sum_{n=2}^{\infty} \frac{1}{K_{n}^{p+r_{n+1}-1}}\left(\left(1+D^{2}\right) K_{n-1}^{1-\frac{1}{p+r_{n-1}}}\right)^{p+r_{n+1}} \\
& \leq \frac{\left(1+D^{2}\right)^{p+r_{1}}}{p-1} \sum_{n=2}^{\infty} \frac{K_{n-1}^{p+r_{n+1}-\frac{p+r_{n+1}}{p+r_{n-1}}}}{K_{n}^{p+r_{n+1}-1}} \\
& =\frac{\left(1+D^{2}\right)^{p+r_{1}}}{p-1} \sum_{n=2}^{\infty}\left(\frac{K_{n-1}}{K_{n}}\right)^{p+r_{n+1}-1} K_{n-1}^{\frac{r_{n-1}-r_{n+1}}{p+r_{n-1}}}
\end{aligned}
$$

Since $K_{n}$ is non-decreasing sequence of positive integers we have by (iii)

$$
\begin{aligned}
K_{n-1}^{\frac{r_{n-1}-r_{n+1}}{p+r_{n-1}}} & \leq K_{n-1}^{r_{n-1}-r_{n+1}} \\
& \leq K_{n-1}^{r_{n-1}-r_{n}} K_{n-1}^{r_{n}-r_{n+1}} \\
& \leq K_{n-1}^{r_{n-1}-r_{n}} K_{n}^{r_{n}-r_{n+1}} \\
& \leq D^{2} .
\end{aligned}
$$


Moreover, we obtain by (iv)

$$
\left(\frac{K_{n-1}}{K_{n}}\right)^{1-\frac{1}{p+r_{n}}} \leq \frac{D}{n-1}
$$

and so,

$$
\left(\frac{K_{n-1}}{K_{n}}\right)^{p+r_{n}-1} \leq \frac{D^{p+r_{n}}}{(n-1)^{p+r_{n}}} \leq \frac{D^{p+r_{1}}}{(n-1)^{p+r_{n}}} .
$$

Since $r_{n} \searrow 0$ we have $\frac{p+r_{n+1}-1}{p+r_{n}-1} \rightarrow 0$ and sonsequently,

$$
\begin{aligned}
\sum_{n=2}^{\infty}\left(\frac{K_{n-1}}{K_{n}}\right)^{p+r_{n+1}-1} & =\sum_{n=2}^{\infty}\left(\frac{K_{n-1}}{K_{n}}\right)^{\left(p+r_{n}-1\right) \frac{p+r_{n+1}-1}{p+r_{n}-1}} \\
& \leq D^{p+r_{1}} \sum_{n=2}^{\infty}(n-1)^{\left(p+r_{n}-1\right) \frac{p+r_{n+1}-1}{p+r_{n}-1}} \\
& :=\widetilde{D}<\infty
\end{aligned}
$$

which gives with (4.6) and (4.7)

$$
I_{1} \leq \frac{\left(1+D^{2}\right)^{p+r_{1}}}{p-1} D^{2} \widetilde{D} .
$$

Estimate $I_{3}$. Fix for a moment $n$. Denote $q=p+r_{n}$. Since $\mathfrak{a}^{(n)} \in \ell^{p+r_{n}}=\ell^{q}$ we have by Theorem 2.7 with a constant $C_{p}:=C_{p, p+r_{0}}$

$$
\begin{aligned}
\sum_{j \in \mathcal{K}_{n}}\left(M \mathfrak{a}^{(n)}\right)_{j}^{p+r_{n}} & =\sum_{j \in \mathcal{K}_{n}}\left(M \mathfrak{a}^{(n)}\right)_{j}^{q} \\
& \leq \sum_{j \in \mathbb{Z}}\left(M \mathfrak{a}^{(n)}\right)_{j}^{q} \leq C_{p} \sum_{j \in \mathbb{Z}}\left(\mathfrak{a}^{(n)}\right)_{j}^{q} \\
& =C_{p} \sum_{j \in \mathcal{K}_{n}}\left(\mathfrak{a}^{(n)}\right)_{j}^{p+r_{n}}
\end{aligned}
$$

which gives

$$
\begin{aligned}
I_{3} & =\sum_{n=1}^{\infty} \sum_{j \in \mathcal{K}_{n}}\left(M^{-} \mathfrak{a}^{(n)}\right)_{j}^{p+r_{n}} \\
& \leq \sum_{n=1}^{\infty} \sum_{j \in \mathcal{K}_{n}}\left(M \mathfrak{a}^{(n)}\right)_{j}^{p+r_{n}}=\sum_{n=1}^{\infty} C_{p} \sum_{j \in \mathcal{K}_{n}}\left(\mathfrak{a}^{(n)}\right)_{j}^{p+r_{n}} \\
& =C_{p} \sum_{j \in \mathbb{Z}} a_{j}^{p_{j}} \leq C_{p}<\infty .
\end{aligned}
$$


Let us estimate $I_{2}$. Define a sequence $\mathfrak{b} \in \mathcal{M}$ by

$$
b_{j}= \begin{cases}\left(M^{-} \mathfrak{a}^{(n)}\right)_{j} & \text { if } j \in \mathcal{K}_{n+1} \quad n=1,2, \ldots \\ 0 & \text { if } j \leq A_{1} .\end{cases}
$$

Fix for a moment $n$ and set $q=p+r_{n}$. Since $\mathfrak{a}^{(n)} \in \ell^{p+r_{n}}=\ell^{q}$ with constant exponent sequence we obtain by Theorem 2.7 (again with the constant $C_{p}:=C_{p, p+r_{0}}$ )

$$
\begin{aligned}
\sum_{j \in \mathcal{K}_{n+1}} b_{j}^{p+r_{n}} & =\sum_{j \in \mathcal{K}_{n+1}}\left(\left(M^{-} \mathfrak{a}^{(n)}\right)_{j}\right)^{p+r_{n}} \\
& \leq \sum_{j \in \mathcal{K}_{n+1}}\left(\left(M^{-} \mathfrak{a}^{(n)}\right)_{j}\right)^{q} \\
& \leq \sum_{j \in \mathbb{Z}}\left(\left(M \mathfrak{a}^{(n)}\right)_{j}\right)^{q} \leq C_{p} \sum_{j \in \mathbb{Z}}\left(\mathfrak{a}^{(n)}\right)_{j}^{q} \\
& =C_{p} \sum_{j \in \mathcal{K}_{n}}\left(\mathfrak{a}^{(n)}\right)_{j}^{p+r_{n}} \\
& =C_{p} \sum_{j \in \mathcal{K}_{n}} a_{j}^{p+r_{n}}
\end{aligned}
$$

and so, using the definition of $\mathfrak{q}$ from (i) we have by (4.1)

$$
\sum_{j \in \mathbb{Z}} b_{j}^{q_{j}}=\sum_{n=1}^{\infty} \sum_{j \in \mathcal{K}_{n+1}} b_{j}^{p+r_{n}} \leq C_{p} \sum_{n=1}^{\infty} \sum_{j \in \mathcal{K}_{n}} a_{j}^{p+r_{n}}=C_{p} \sum_{j \in \mathbb{Z}} a_{j}^{p_{j}} \leq C_{p} .
$$

By (i) we have $\mathfrak{q}-\mathfrak{p} \in \mathcal{P}$ and so, by Lemma 2.12 we obtain

$$
\sum_{j \in \mathbb{Z}} b_{j}^{p_{j}} \leq \max \left\{1, \sup _{j \in \mathbb{Z}} C_{p}^{\frac{p_{j}}{q_{j}}}\right\} C
$$

which gives

$$
\begin{aligned}
I_{2} & =\sum_{n=1}^{\infty} \sum_{j \in \mathcal{K}_{n+1}}\left(M^{-} \mathfrak{a}^{(n)}\right)_{j}^{p+r_{n+1}} \\
& =\sum_{n=1}^{\infty} \sum_{j \in \mathcal{K}_{n+1}} b_{j}^{p+r_{n+1}} \\
& =\sum_{j \in \mathbb{Z}} b_{j}^{p_{j}} \leq \max \left\{1, \sup _{j \in \mathbb{Z}} C_{p}^{\frac{p_{j}}{q_{j}}}\right\} C .
\end{aligned}
$$


Finally, using (4.8), (4.9) and (4.10) we have

$$
\begin{aligned}
\sum_{j \in \mathbb{Z}}\left(M^{-} \mathfrak{a}\right)_{j}^{p_{j}} & \leq 3^{p+r_{1}}\left(I_{1}+I_{2}+I_{3}\right) \\
& \leq 3^{p+r_{1}}\left(\frac{\left(1+D^{2}\right)^{p+r_{1}}}{p-1} D^{2} \widetilde{D}+C_{p}+\max \left\{1, \sup _{j \in \mathbb{Z}} C_{p}^{\frac{p_{j}}{q_{j}}}\right\} C\right)
\end{aligned}
$$

which finishes the proof.

Lemma 4.2. Let a real number $D$ satisfy the assumption (iv) from Lemma 4.1. Then there is a constant $C>0$ such that the implication

$$
\sum_{j=1}^{\infty} a_{j}^{p_{j}} \leq 1 \Rightarrow \sum_{j=1}^{\infty}\left(M^{+} \mathfrak{a}\right)_{j}^{p_{j}} \leq C
$$

holds for all $\mathfrak{a} \in \mathcal{M}_{+}$with $a_{j}=0$ for $j \leq 0$ and the implication

$$
\sum_{j=-\infty}^{-1} a_{j}^{p_{j}} \leq 1 \Rightarrow \sum_{j=-\infty}^{-1}\left(M^{-} \mathfrak{a}\right)_{j}^{p_{j}} \leq C
$$

holds for all $\mathfrak{a} \in \mathcal{M}_{+}$with $a_{j}=0$ for $j \geq 0$.

Proof. Since $\mathfrak{p}$ is symmetric it suffices to prove only the first implication. Assume

$$
\sum_{j=1}^{\infty} a_{j}^{p_{j}} \leq 1
$$

Fix $n \in \mathbb{N}$ and $j \in \mathcal{K}_{n}$. Denote $\mathcal{L}_{k}=\bigcup_{s=k}^{\infty} \mathcal{K}_{s}$ for any $k \in \mathbb{N}$. Then $\mathfrak{a}=\sum_{s=1}^{n+1} \mathfrak{a} \chi_{\mathcal{K}_{s}}+\mathfrak{a} \chi_{\mathcal{L}_{n+2}}$. Since $\left(M^{+} \mathfrak{a} \chi_{\mathcal{K}_{s}}\right)_{j}=0$ for $s<n$ we have by the sub-linearity of $M^{+}$

$$
\left(M^{+} \mathfrak{a}\right)_{j} \leq\left(M^{+} \mathfrak{a} \chi_{\mathcal{K}_{n}}\right)_{j}+\left(M^{+} \mathfrak{a} \chi_{\mathcal{K}_{n+1}}\right)_{j}+\left(M^{+} \mathfrak{a} \chi_{\mathcal{L}_{n+2}}\right)_{j} .
$$

Estimate $\left(M^{+} \mathfrak{a} \chi_{\mathcal{L}_{n+2}}\right)_{j}$. Clearly,

$$
\left(M^{+} \mathfrak{a} \chi_{\mathcal{L}_{n+2}}\right)_{j} \leq \sup _{k>A_{n+1}} \frac{1}{k-j+1} \sum_{j=1+A_{n+1}}^{k} a_{j}
$$

Since $\sum_{j=1}^{\infty} a_{j} \leq 1$ we have $a_{j} \leq 1$ and so, $a_{j}^{p+r_{n}} \leq a_{j}^{p_{j}}$. By Jensen's inequality we obtain

$$
\begin{aligned}
\left(M^{+} \mathfrak{a} \chi_{\mathcal{L}_{n+2}}\right)_{j}^{p+r_{n}} & \leq\left(\sup _{k>A_{n+1}} \frac{1}{k-j+1} \sum_{j=1+A_{n+1}}^{k} a_{j}\right)^{p+r_{n}} \\
& \leq \sup _{k>A_{n+1}} \frac{1}{k-j+1} \sum_{j=1+A_{n+1}}^{k} a_{j}^{p+r_{n}}
\end{aligned}
$$




$$
\begin{aligned}
& \leq \sup _{k>A_{n+1}} \frac{1}{k-j+1} \sum_{j=1+A_{n+1}}^{k} a_{j}^{p_{j}} \\
& \leq \sup _{k>A_{n+1}} \frac{1}{K_{n+1}} \sum_{j=1+A_{n+1}}^{k} a_{j}^{p_{j}} \\
& \leq \frac{1}{K_{n+1}} .
\end{aligned}
$$

Thus, by (4.12) we have

$$
\begin{aligned}
& \sum_{j \in \mathcal{K}_{n}}\left(M^{+} \mathfrak{a}\right)_{j}^{p+r_{n}} \\
& \leq 3^{p+r_{n}-1}\left\{\sum_{j \in \mathcal{K}_{n}}\left(M^{+} \mathfrak{a} \chi_{\mathcal{K}_{n}}\right)_{j}^{p+r_{n}}+\sum_{j \in \mathcal{K}_{n}}\left(M^{+} \mathfrak{a} \chi_{\mathcal{K}_{n+1}}\right)_{j}^{p+r_{n}}+\sum_{j \in \mathcal{K}_{n}} \frac{1}{K_{n+1}}\right\} \\
& =3^{p+r_{n}-1}\left(I_{1}+I_{2}+I_{3}\right) .
\end{aligned}
$$

Estimate $I_{1}$ and $I_{2}$. By Lemma 2.7 with a constant $C_{p}:=C_{p, p+r_{0}}$ we obtain

$$
\begin{aligned}
I_{1} & =\sum_{j \in \mathcal{K}_{n}}\left(M^{+}\left(\mathfrak{a} \chi_{\mathcal{K}_{n}}\right)\right)_{j}^{p+r_{n}} \\
& \leq \sum_{j \in \mathbb{Z}}\left(M\left(\mathfrak{a} \chi_{\mathcal{K}_{n}}\right)\right)_{j}^{p+r_{n}} \\
& \leq C_{p} \sum_{j \in \mathbb{Z}}\left(\mathfrak{a} \chi_{\mathcal{K}_{n}}\right)_{j}^{p+r_{n}} \\
& =C_{p} \sum_{j \in \mathcal{K}_{n}} a_{j}^{p+r_{n}}
\end{aligned}
$$

and analogously,

$$
\begin{aligned}
I_{2} & =\sum_{j \in \mathcal{K}_{n}}\left(M^{+}\left(\mathfrak{a} \chi_{\mathcal{K}_{n+1}}\right)\right)_{j}^{p+r_{n}} \\
& \leq \sum_{j \in \mathbb{Z}}\left(M\left(\mathfrak{a} \chi_{\mathcal{K}_{n+1}}\right)\right)_{j}^{p+r_{n}} \\
& \leq C_{p} \sum_{j \in \mathbb{Z}}\left(\mathfrak{a} \chi_{\mathcal{K}_{n+1}}\right)_{j}^{p+r_{n}} \\
& =C_{p} \sum_{j \in \mathcal{K}_{n+1}} a_{j}^{p+r_{n}} .
\end{aligned}
$$


Since $a_{j} \leq 1$ and $r_{n} \geq r_{n+1}$ for $n \geq 0$ we have $a_{j}^{p+r_{n}} \leq a_{j}^{p+r_{n+1}}$ which gives with (4.15)

$$
I_{2} \leq C_{p} \sum_{j \in \mathcal{K}_{n+1}} a_{j}^{p+r_{n}} \leq C_{p} \sum_{j \in \mathcal{K}_{n+1}} a_{j}^{p+r_{n+1}} .
$$

Then

$$
\begin{aligned}
& \sum_{j \in \mathcal{K}_{n}}\left(M^{+} \mathfrak{a}\right)_{j}^{p+r_{n}} \\
& \leq 3^{p+r_{n}-1}\left\{C_{p} \sum_{j \in \mathcal{K}_{n}} a_{j}^{p+r_{n}}+C_{p} \sum_{j \in \mathcal{K}_{n+1}} a_{j}^{p+r_{n+1}}+\frac{K_{n}}{K_{n}+1}\right\} \\
& \leq 3^{p+r_{0}-1}\left\{C_{p} \sum_{j \in \mathcal{K}_{n}} a_{j}^{p+r_{n}}+C_{p} \sum_{j \in \mathcal{K}_{n+1}} a_{j}^{p+r_{n+1}}+\frac{K_{n}}{K_{n+1}}\right\}
\end{aligned}
$$

which yields immediately with (4.14) and (4.13)

$$
\begin{aligned}
\sum_{j=1}^{\infty}\left(M^{+} \mathfrak{a}\right)_{j}^{p_{j}}= & \sum_{n=1}^{\infty} \sum_{j \in \mathcal{K}_{n}}\left(M^{+} \mathfrak{a}\right)_{j}^{p_{j}} \\
\leq & 3^{p+r_{1}-1} C_{p} \sum_{n=1}^{\infty} \sum_{j \in \mathcal{K}_{n}} a_{j}^{p+r_{n}}+3^{p+r_{0}-1} C_{p} \sum_{n=1}^{\infty} \sum_{j \in \mathcal{K}_{n+1}} a_{j}^{p+r_{n+1}} \\
& \quad+3^{p+r_{1}-1} \sum_{n=1}^{\infty} \frac{K_{n}}{K_{n+1}} \\
\leq & 3^{p+r_{1}-1} \max \left\{1, C_{p}\right\}\left(2 \sum_{j=1}^{\infty} a_{j}^{p_{j}}+\sum_{n=1}^{\infty} \frac{K_{n}}{K_{n+1}}\right) \\
\leq & 3^{p+r_{1}-1} \max \left\{1, C_{p}\right\}\left(2+\sum_{n=1}^{\infty} \frac{K_{n}}{K_{n+1}}\right) .
\end{aligned}
$$

Now, by the assumption $n K_{n}^{1-\frac{1}{p+r_{n+1}}} \leq D K_{n+1}^{1-\frac{1}{p+r_{n+1}}}$ we have

$$
\left(\frac{K_{n}}{K_{n+1}}\right)^{1-\frac{1}{p+r_{n+1}}} \leq \frac{D}{n}
$$

which gives

$$
\left(\frac{K_{n}}{K_{n+1}}\right) \leq\left(\frac{D}{n}\right)^{\frac{p+r_{n+1}}{p+r_{n+1}-1}} \leq \frac{D^{\frac{p}{p-1}}}{n^{\frac{p+r_{1}}{p+r_{1}-1}}} .
$$


Since $\frac{p+r_{1}}{p+r_{1}-1}>1$ the series $\sum_{n=1}^{\infty} \frac{K_{n}}{K_{n+1}}$ converges and so, by (4.16) we obtain

$$
\sum_{j=1}^{\infty}\left(M^{+} \mathfrak{a}\right)_{j}^{p_{j}} \leq 3^{p+r_{1}-1} \max \left\{1, C_{p}\right\}\left(2+\sum_{n=1}^{\infty} \frac{K_{n}}{K_{n+1}}\right):=C
$$

which proves the lemma.

Lemma 4.3. Let $\mathfrak{p}$ satisfy the assumptions (i)-(iv) from Lemma 4.1. Then there is $C>0$ such that the implication

$$
\sum_{j=1}^{\infty} a_{j}^{p_{j}} \leq 1 \Rightarrow \sum_{j=-\infty}^{-1}\left(M^{+} \mathfrak{a}\right)_{j}^{p_{j}} \leq C
$$

holds for all $\mathfrak{a} \in \mathcal{M}_{+}, a_{j}=0$ for $j \leq 0$ and the implication

$$
\sum_{j=-\infty}^{-1} a_{j}^{p_{j}} \leq 1 \Rightarrow \sum_{j=1}^{\infty}\left(M^{-} \mathfrak{a}\right)_{j}^{p_{j}} \leq C
$$

holds for all $\mathfrak{a} \in \mathcal{M}_{+}, a_{j}=0$ for $j \geq 0$.

Proof. Since $\mathfrak{p}$ is symmetric it suffices to prove only the first part of the lemma. Assume $\mathfrak{a} \in \mathcal{M}_{+}, \sum_{j=1}^{\infty} a_{j}^{p_{j}} \leq 1$ and $a_{j}=0$ for $j \leq 0$. Fix an integer $j<0$. Find an integer $k>0$ such that

$$
\left(M^{+} \mathfrak{a}\right)_{j} \leq \frac{2}{k-j+1} \sum_{s=1}^{k} a_{s} .
$$

If $k \geq-j$ then evidently $-j$ lies between 1 and $k$ and so,

$$
\frac{2}{k-j+1} \sum_{s=1}^{k} a_{s} \leq \frac{2}{k} \sum_{s=1}^{k} a_{s} \leq 2(M \mathfrak{a})_{-j} .
$$

If $k<-j$ then

$$
\frac{2}{k-j+1} \sum_{s=1}^{k} a_{s} \leq \frac{2}{-j} \sum_{s=1}^{k} a_{s} \leq \frac{2}{-j} \leq 2(M \mathfrak{a})_{-j} .
$$

In both cases we obtain

$$
\left(M^{+} \mathfrak{a}\right)_{j} \leq 2(M \mathfrak{a})_{-j}
$$

Denote by $C_{1}$ the constant $C$ from Lemma 4.1 and by $C_{2}$ the constant $C$ from Lemma 4.2. Now, using Lemma 2.4, Lemma 4.1, Lemma 4.2 and the symmetry of $\mathfrak{p}$ we obtain

$$
\sum_{j=-\infty}^{-1}\left(M^{+} \mathfrak{a}\right)_{j}^{p_{j}} \leq \sum_{j=-\infty}^{-1} 2^{p_{j}}(M \mathfrak{a})_{-j}^{p_{j}}
$$




$$
\begin{aligned}
& \leq \sum_{j=-\infty}^{-1} 2^{p_{j}}\left(2\left(\left(M^{-} \mathfrak{a}\right)_{-j}+\left(M^{+} \mathfrak{a}\right)_{-j}\right)\right)^{p_{j}} \\
& \leq \sum_{j=-\infty}^{-1} 4^{p_{j}}\left(\left(M^{-} \mathfrak{a}\right)_{-j}+\left(M^{+} \mathfrak{a}\right)_{-j}\right)^{p_{-j}} \\
& \leq 4^{p+r_{0}} \sum_{j=1}^{\infty}\left(\left(M^{-} \mathfrak{a}\right)_{j}+\left(M^{+} \mathfrak{a}\right)_{j}\right)^{p_{j}} \\
& \leq 4^{p+r_{0}} \sum_{j=1}^{\infty} 2^{p_{j}-1}\left(\left(M^{-} \mathfrak{a}\right)_{j}^{p_{j}}+\left(M^{+} \mathfrak{a}\right)_{j}^{p_{j}}\right) \\
& \leq 4^{p+r_{0}} 2^{p+r_{0}-1}\left(C_{1}+C_{2}\right):=C
\end{aligned}
$$

which finishes the proof.

Lemma 4.4. Let $\mathfrak{p}$ satisfy the assumptions (i)-(iv) from Lemma 4.1. Then there exists $C>0$ such that implications

$$
\sum_{j \in \mathbb{Z}} a_{j}^{p_{j}} \leq 1 \Rightarrow \sum_{j \in \mathbb{Z}}\left(M^{-} a\right)_{j}^{p_{j}} \leq C
$$

and

$$
\sum_{j \in \mathbb{Z}} a_{j}^{p_{j}} \leq 1 \Rightarrow \sum_{j \in \mathbb{Z}}\left(M^{+} a\right)_{j}^{p_{j}} \leq C
$$

hold for all $\mathfrak{a} \in \mathcal{M}$.

Proof. Let $C_{1}, C_{2}, C_{3}$ denote the constants $C$ from Lemmas 4.1, 4.2 and 4.3. Since $\mathfrak{p}$ is symmetric it suffices to prove only the first implication. Assume $\sum_{j \in \mathbb{Z}} a_{j}^{p_{j}} \leq 1$. Denote $\mathfrak{a}_{+}=\mathfrak{a} \chi_{\{1,2, \ldots\}}, \mathfrak{a}_{-}=\mathfrak{a} \chi_{\{-1,-2, \ldots\}}$ and $\mathfrak{a}_{0}=\mathfrak{a} \chi_{\{0\}}$. Clearly, $\mathfrak{a}=\mathfrak{a}_{-}+\mathfrak{a}_{0}+\mathfrak{a}_{+}$and due to the sub-linearity of $M^{-}$ we obtain

$$
\left(M^{-} \mathfrak{a}\right)_{j} \leq\left(M^{-}\left(\mathfrak{a}_{-}\right)\right)_{j}+\left(M^{-}\left(\mathfrak{a}_{0}\right)\right)_{j}+\left(M^{-}\left(\mathfrak{a}_{+}\right)\right)_{j} .
$$

Thus,

$$
\begin{aligned}
\sum_{j \in \mathbb{Z}}\left(M^{-} \mathfrak{a}\right)_{j}^{p_{j}} \leq & \sum_{j \in \mathbb{Z}} 3^{p_{j}-1}\left(\left(M^{-}\left(\mathfrak{a}_{-}\right)\right)_{j}^{p_{j}}+\left(M^{-}\left(\mathfrak{a}_{0}\right)\right)_{j}^{p_{j}}+\left(M^{-}\left(\mathfrak{a}_{+}\right)\right)_{j}^{p_{j}}\right) \\
\leq & 3^{p+r_{0}-1}\left(\sum_{j \in \mathbb{Z}}\left(M^{-}\left(\mathfrak{a}_{-}\right)\right)_{j}^{p_{j}}+\sum_{j \in \mathbb{Z}}\left(M^{-}\left(\mathfrak{a}_{0}\right)\right)_{j}^{p_{j}}\right. \\
& \left.\quad+\sum_{j \in \mathbb{Z}}\left(M^{-}\left(\mathfrak{a}_{+}\right)\right)_{j}^{p_{j}}\right) \\
= & 3^{p+r_{0}-1}\left(I_{1}+I_{2}+I_{3}\right) .
\end{aligned}
$$


Estimate $I_{3}$. Since $\left(\mathfrak{a}_{+}\right)_{j}=0$ for $j \leq 0$ we have $\left(M^{-}\left(\mathfrak{a}_{+}\right)\right)_{j}=0$ for $j \leq 0$ and so,

$$
I_{3}=\sum_{j \in \mathbb{Z}}\left(M^{-}\left(\mathfrak{a}_{+}\right)\right)_{j}^{p_{j}}=\sum_{j=1}^{\infty}\left(M^{-}\left(\mathfrak{a}_{+}\right)\right)_{j}^{p_{j}}
$$

Clearly, $\sum_{j=1}^{\infty} a_{j}^{p_{j}}=\sum_{j \in \mathbb{Z}}\left(\mathfrak{a}_{+}\right)_{j}^{p_{j}} \leq \sum_{j \in \mathbb{Z}}(\mathfrak{a})_{j}^{p_{j}} \leq 1$ and by Lemma 4.1 we obtain

$$
I_{3}=\sum_{j \in \mathbb{Z}}\left(M^{-}\left(\mathfrak{a}_{+}\right)\right)_{j}^{p_{j}} \leq C_{1} .
$$

Estimate $I_{1}$. Clearly,

$$
I_{1}=\sum_{j=-\infty}^{-1}\left(M^{-}\left(\mathfrak{a}_{-}\right)\right)_{j}^{p_{j}}+\left(M^{-}\left(\mathfrak{a}_{-}\right)\right)_{0}^{p_{0}}+\sum_{j=1}^{\infty}\left(M^{-}\left(\mathfrak{a}_{-}\right)\right)_{j}^{p_{j}} .
$$

Since $\sum_{j=-\infty}^{-1} a_{j}^{p_{j}} \leq 1$ then $a_{j} \leq 1$ for all $j$ and consequently, $\frac{1}{-k+1} \sum_{s=k}^{0} a_{s} \leq 1$ which immediately yields $\left(M^{-}\left(\mathfrak{a}_{-}\right)\right)_{0} \leq 1$ and so, using (4.19), Lemma 4.2 and Lemma 4.3 we have

$$
I_{1} \leq C_{2}+1+C_{3}
$$

Estimate $I_{2}$. Clearly,

$$
I_{2}=\sum_{j \in \mathbb{Z}}\left(M^{-}\left(\mathfrak{a}_{0}\right)\right)_{j}^{p_{j}}=\sum_{j=0}^{\infty}\left(M^{-}\left(\mathfrak{a}_{0}\right)\right)_{j}^{p_{j}} .
$$

Fix for a moment $j \geq 0$. Then using the fact $a_{0} \leq 1$ we have $\left(M^{-}\left(\mathfrak{a}_{0}\right)\right)_{j}=$ $\frac{a_{0}}{1+j} \leq \frac{1}{1+j}$. Then

$$
I_{2} \leq \sum_{j=0}^{\infty} \frac{a_{0}^{p_{j}}}{(1+j)^{p_{j}}} \leq \sum_{j=0}^{\infty} \frac{1}{(1+j)^{p_{j}}} \leq \sum_{j=0}^{\infty} \frac{1}{(1+j)^{p}} .
$$

Thus, (4.18), (4.18), (4.20) and (4.21) give

$$
\sum_{j \in \mathbb{Z}}\left(M^{-} \mathfrak{a}\right)_{j}^{p_{j}} \leq 3^{p+r_{0}-1}\left(C_{1}+C_{2}+1+C_{3}+\sum_{j=0}^{\infty} \frac{1}{(1+j)^{p}}\right):=C
$$

which finishes the proof. 
Proposition 4.5. Let $\mathfrak{p}$ satisfy the assumptions (i)-(iv) from Lemma 4.1. Then $M$ is bounded on $\ell^{\mathfrak{p}}$.

Proof. The proof is an immediate consequence of Lemma 2.4 and Lemma 4.4 .

\section{Examples}

Assume in this section $\alpha, \beta>0$ and $L>1$. Let [.] denote the integer part as usually. Set $K_{n}=\left[L^{n^{\alpha}}\right]$ and $r_{n}=\frac{1}{n^{\beta}}$ for $n \in \mathbb{N}$ and $r_{0}=1$. Let $\mathfrak{p}$ be given by (2.3). Evidently,

$$
\lim _{|n| \rightarrow \infty} p_{n}=p
$$

and so, $\mathfrak{p} \in \mathcal{B}_{p}$.

Proposition 5.1. Let $\alpha \leq \beta$. Then $M$ is bounded on $\ell^{\mathfrak{p}}$.

Proof. Choose $c=\frac{1}{2 L}$ in Definition 2.9 and we have

$$
\begin{aligned}
\sum_{n \in \mathbb{Z} ;\left|p_{n}-p\right|>0}\left|p_{n}-p\right| c^{\frac{1}{p_{n}-p \mid}} & =2 \sum_{n=1}^{\infty} K_{n} r_{n} c^{\frac{1}{r_{n}}} \\
& =2 \sum_{n=1}^{\infty}\left[L^{n^{\alpha}}\right] \frac{1}{n^{\beta}}\left(\frac{1}{2 L}\right)^{n^{\beta}} \\
& \leq 2 \sum_{n=1}^{\infty} L^{n^{\alpha}} \frac{1}{n^{\beta} 2^{n^{\beta}}}\left(\frac{1}{L}\right)^{n^{\beta}} \\
& \leq 2 \sum_{n=1}^{\infty} \frac{L^{n^{\alpha}}-n^{\beta}}{n^{\beta}} \frac{1}{2^{n^{\beta}}} .
\end{aligned}
$$

According to the assumption we have $n^{\alpha} \leq n^{\beta}$ and so, $L^{n^{\alpha}-n^{\beta}} \leq 1$. Since $\beta>0$ we obtain

$$
\sum_{n \in \mathbb{Z} ;\left|p_{n}-p\right|>0}\left|p_{n}-p\right| c^{\frac{1}{\left|p_{n}-p\right|}} \leq 2 \sum_{n=1}^{\infty} \frac{1}{2^{n^{\beta}}}<\infty
$$

which proves $\mathfrak{p} \in \mathcal{P}_{p}$ and consequently, by Lemma $2.13, M$ is bounded.

We shall in the next frequently use the following easy estimate which can be obtained using Lagrange mean value theorem. For $\beta>0$ and $n \in \mathbb{N}$ we have

$$
\frac{\beta}{(n+1)^{1+\beta}} \leq \frac{1}{n^{\beta}}-\frac{1}{(n+1)^{\beta}} \leq \frac{\beta}{n^{1+\beta}} .
$$


Proposition 5.2. Let $\alpha>1+\beta$. Then $M$ is not bounded on $\ell^{\mathfrak{p}}$.

Proof. Since $L^{n^{\alpha}} \nearrow \infty$ we can choose a real $L_{1}, 1<L_{1}<L$, and we have

$$
\left[L^{n^{\alpha}}\right] \geq L^{n^{\alpha}}-1 \geq L_{1}^{n^{\alpha}}
$$

for sufficient large $n$. Then

$$
K_{n}^{r_{n}-r_{n+1}}=\left[L^{n^{\alpha}}\right]^{\frac{1}{n^{\beta}}-\frac{1}{(n+1)^{\beta}}} \geq L_{1}^{n^{\alpha} \frac{\beta}{(n+1)^{1+\beta}}} .
$$

By the assumption $\alpha>1+\beta$ we have $\frac{n^{\alpha}}{(n+1)^{\beta}} \nearrow \infty$ and consequently, $K_{n}^{r_{n}-r_{n+1}}$ is unbounded. The assertion of our lemma now follows from Proposition 3.1.

Lemma 5.3. Let $\alpha \leq 1+\beta$. Then $\mathfrak{p}$ satisfies the assumption (i) from Lemma 4.1 .

Proof. Prove $\mathfrak{q}-\mathfrak{p} \in \mathcal{P}$. Choose $c=L^{-\beta}$ in Definition 2.9. Clearly,

$$
\begin{aligned}
A & :=\sum_{n \in \mathbb{Z} ;\left|q_{n}-p_{n}\right|>0}\left|q_{n}-p_{n}\right| c^{\frac{1}{q_{n}-p_{n}}} \\
& =2 \sum_{n=1}^{\infty} K_{n}\left(r_{n-1}-r_{n}\right) L^{\frac{\beta}{r_{n-1}-r_{n}}} \\
& =\underbrace{2 K_{1}\left(r_{0}-r_{1}\right) L^{\frac{\beta}{r_{0}-r_{1}}}}_{B}+2 \sum_{n=2}^{\infty}\left[L^{n^{\alpha}}\right]\left(\frac{1}{(n-1)^{\beta}}-\frac{1}{n^{\beta}}\right) L^{\frac{\beta}{\frac{1}{n-1^{\beta}}-\frac{1}{n^{\beta}}}} .
\end{aligned}
$$

By (5.2) we have

$$
A \leq B+2 \sum_{n=2}^{\infty} L^{n^{\alpha}} \frac{\beta}{(n-1)^{1+\beta}} L^{-\frac{1}{n^{1+\beta}}}=B+2 \beta \sum_{n=2}^{\infty} \frac{L^{n^{\alpha}-n^{1+\beta}}}{(n-1)^{1+\beta}} .
$$

By the assumption $\alpha>1+\beta$ we have $L^{n^{\alpha}-n^{1+\beta}} \leq 1$ and so,

$$
A \leq B+2 \beta \sum_{n=2}^{\infty} \frac{1}{(n-1)^{1+\beta}}<\infty
$$

which finishes the proof.

In the next we will use another fact. Since $L^{n^{\alpha}} \nearrow \infty$ we have $\lim _{n \rightarrow \infty}\left[L^{n^{\alpha}}\right] L^{-n^{\alpha}}=1$ and so, there exists $\omega>1$ such that

$$
L^{n^{\alpha}} \leq \omega\left[L^{n^{\alpha}}\right]
$$

holds for all $n \geq 1$.

Lemma 5.4. Let $\beta \leq \alpha$. Then the property assumption (ii) from Lemma 4.1 is satisfied. 
Proof. Assume $m, n \in \mathbb{N}, m \leq n$. Then the easy inequality $\frac{A+1}{B+1} \leq \frac{A}{B}$ for $A \geq B>0$ gives

$$
\frac{p n^{\beta}+1}{p m^{\beta}+1} \leq \frac{p n^{\beta}}{p m^{\beta}}=\left(\frac{n}{m}\right)^{\beta} \leq\left(\frac{n}{m}\right)^{\alpha} .
$$

Since $\frac{(p-1) m^{\beta}+1}{(p-1) n^{\beta}+1} \leq 1$ we obtain

$$
\frac{(p-1) m^{\beta}+1}{(p-1) n^{\beta}+1} \frac{p n^{\beta}+1}{p m^{\beta}+1} \leq\left(\frac{n}{m}\right)^{\alpha}
$$

which can be rewritten as

$$
\frac{1-\frac{m^{\beta}}{p m^{\beta}+1}}{1-\frac{n^{\beta}}{p n^{\beta}+1}} \leq\left(\frac{n}{m}\right)^{\alpha}
$$

and so,

$$
m^{\alpha}\left(1-\frac{1}{p+\frac{1}{m^{\beta}}}\right) \leq n^{\alpha}\left(1-\frac{1}{p+\frac{1}{n^{\beta}}}\right)
$$

Since $L>1$ we obtain

$$
L^{m^{\alpha}\left(1-\frac{1}{p+\frac{1}{m^{\beta}}}\right)} \leq L^{n^{\alpha}\left(1-\frac{1}{p+\frac{1}{n^{\beta}}}\right)} .
$$

and using (5.3) we have

$$
\begin{aligned}
K_{m}^{1-\frac{1}{p+r_{m}}} & =\left[L^{m^{\alpha}}\right]^{1-\frac{1}{p+\frac{1}{m^{\beta}}}} \\
& \leq L^{m^{\alpha}\left(1-\frac{1}{p+\frac{1}{m^{\beta}}}\right)} \\
& \leq L^{n^{\alpha}\left(1-\frac{1}{p+\frac{1}{n^{\beta}}}\right)} \\
& \leq\left(\omega\left[L^{n^{\alpha}}\right]\right)^{1-\frac{1}{p+\frac{1}{n^{\beta}}}} \\
& \leq \omega\left[L^{n^{\alpha}}\right]^{1-\frac{1}{p+\frac{1}{n^{\beta}}}} \\
& =\omega K_{n}^{1-\frac{1}{p+r_{n}}}
\end{aligned}
$$

which finishes the proof.

Lemma 5.5. Let $\alpha \leq 1+\beta$. Then the assumption (iii) from Lemma 4.1 is satisfied.

Proof. By (5.2) we can write

$$
\left.K_{n}^{r_{n}-r_{n-1}}=\left[L^{n^{\alpha}}\right]^{\left(\frac{1}{n^{\beta}}-\frac{1}{(n+1)^{\beta}}\right.}\right) \leq L^{n^{\alpha} \frac{\beta}{n^{1+\beta}}}=\left(L^{\beta}\right)^{n^{\alpha-\beta-1}} \leq L^{\beta}
$$

which proves the lemma. 
Lemma 5.6. Let $\alpha>1$. Then the the assumption (iv) from Lemma 4.1 is satisfied.

Proof. Set $S=L^{1-\frac{1}{p}}$. Since $\frac{K_{n}}{K_{n+1}} \leq 1$ and $S>1$ we have by (5.3)

$$
\begin{aligned}
n\left(\frac{K_{n}}{K_{n+1}}\right)^{1-\frac{1}{p+r_{n+1}}} & \leq n\left(\frac{K_{n}}{K_{n+1}}\right)^{1-\frac{1}{p}} \\
& =n\left(\frac{\left[L^{n^{\alpha}}\right]}{\left[L^{(n+1)^{\alpha}}\right]}\right)^{1-1 / p} \\
& \leq n\left(\frac{\omega L^{n^{\alpha}}}{L^{(n+1)^{\alpha}}}\right)^{1-1 / p} \\
& =\omega^{1-1 / p} n S^{n^{\alpha}-(n+1)^{\alpha}} .
\end{aligned}
$$

By Lagrange mean value theorem we have $(n+1)^{\alpha}-n^{\alpha} \geq \alpha n^{\alpha-1}$. Since $\alpha>1$ we obtain $n S^{n^{\alpha}-(n+1)^{\alpha}} \leq n S^{-\alpha n^{\alpha-1}} \rightarrow 0$ for $n \rightarrow \infty$. Thus, $n\left(\frac{K_{n}}{K_{n+1}}\right)^{1-\frac{1}{p+r_{n+1}}}$ is bounded which proves the lemma.

Proposition 5.7. Let $\beta<\alpha \leq 1+\beta$. Then $M$ is bounded on $\ell^{\mathfrak{p}}$.

Proof. The assumptions (i), (ii), (iii) and (iv) from Lemma 4.1 are satisfied by Lemmas 5.3, 5.4, 5.5 and 5.6. By Proposition 4.5 $M$ is bounded.

Lemma 5.8. Let $\beta=1$. Then $M$ is bounded on $\ell^{\mathfrak{p}}$ if and only if $0<\alpha \leq 2$.

Proof. Recall that $0<\alpha<\infty$. If $0<\alpha \leq 1$ then $M$ is bounded by Proposition 5.1. If $1<\alpha \leq 2$ then $M$ is bounded by Proposition 5.7. Finally, if $2<\alpha$ then $M$ is unbounded by Proposition (5.2) which finishes the proof.

Theorem 5.9. There exists $\mathfrak{p} \in \mathcal{B}_{p} \backslash \mathcal{P}_{p}$ such that $M$ is unbounded on $\ell^{\mathfrak{p}}$.

Proof. Choose $\alpha>2$ and $\beta=1$. By (5.1) $\mathfrak{p} \in \mathcal{B}_{p}$. According to Lemma $5.8 M$ is not bounded and of course, by Lemma $2.13 \mathfrak{p} \notin \mathcal{P}_{p}$.

Theorem 5.10. There exists $\mathfrak{p} \in \mathcal{B}_{p} \backslash \mathcal{P}_{p}$ such that $M$ is bounded on $\ell^{\mathfrak{p}}$.

Proof. Choose $\beta=1$ and $1<\alpha \leq 2$. By Lemma $5.8 M$ is bounded. By (5.1) we immediately have $\mathfrak{p} \in \mathcal{B}_{p}$. It remains to prove $\mathfrak{p} \notin \mathcal{P}_{p}$. Let $c>0$ be an arbitrary. Then

$$
\sum_{n \in \mathbb{Z} ;\left|p_{n}-p\right|>0}\left|p_{n}-p\right| c^{\frac{1}{\left|p_{n}-p\right|}}=2 \sum_{n=1}^{\infty} K_{n} r_{n} c^{\frac{1}{r_{n}}}=2 \sum_{n=1}^{\infty}\left[L^{n^{\alpha}}\right] \frac{1}{n} c^{n}=\infty .
$$

Thus, $\mathfrak{p} \notin \mathcal{P}_{p}$. 


\section{Boundedness of the maximal operator on $L^{p(\cdot)}(\mathbb{R})$ for a special function $p(x)$}

We consider in this section the Hardy-Littlewood maximal operator on functions and so, we must distinguish it from the maximal operator $M$ on sequences. We will denote the maximal operator on functions by the symbol $\mathfrak{M}$ as in Section 2 .

Definition 6.1. Let $f \in L_{l o c}^{1}\left(\mathbb{R}^{n}\right)$. Set

$$
\mathfrak{M} f(x)=\sup _{Q \ni x} \frac{1}{|Q|} \int_{Q} f(t) d t .
$$

Denote by $\mathfrak{B}\left(\mathbb{R}^{n}\right)$ a set of all bounded measurable functions $p(),. 1 \leq$ ess $\inf \{p(t) ; t \in \mathbb{R}\}$.

Recall some definitions and basic assertions from [2] and [21].

Definition 6.2. Let $p(.) \in \mathfrak{B}\left(\mathbb{R}^{n}\right)$. Say that $p(.) \in \mathcal{L}$ if there is a constant $K>0$ such that

$$
|p(x)-p(y)| \leq \frac{K}{-\ln |x-y|}
$$

for $x, y \in \mathbb{R}^{n}, 0<|x-y| \leq \frac{1}{2}$.

Definition 6.3. Let $\varepsilon($.$) be a measurable function. Denote \mathbb{P}(\varepsilon())=$. $\left\{x \in \mathbb{R}^{n} ; \varepsilon(x)>0\right\}$. Say that $\varepsilon(.) \in \mathcal{P}$ if there exists a positive real number $c$ such that

$$
\int_{\mathbb{P}(\varepsilon(.))} \varepsilon(x) c^{1 / \varepsilon(x)} \mathrm{d} x<\infty .
$$

Definition 6.4. Let $p(.) \in \mathfrak{B}\left(\mathbb{R}^{n}\right)$. Say that $p(.) \in \mathcal{N}$ if there is a real number $p_{\infty}>1$ such that $\left|p()-.p_{\infty}\right| \in \mathcal{P}$.

The following theorem is the main result in [21].

Theorem 6.5. Let $p(.) \in \mathcal{L} \cap \mathcal{N}, 1<\operatorname{ess} \inf \left\{p(t) ; t \in \mathbb{R}^{n}\right\}$. Then $\mathfrak{M}$ is bounded on $L^{p(x)}\left(\mathbb{R}^{n}\right)$.

Proof. See Theorem 2.14 from [21].

It is not difficult to calculate the following important example.

Example 6.6. Let $1<\inf p(.) \leq \sup p()<.\infty, p$ be a real number, $p>1$, and $p(.) \in \mathcal{L}$.

(i) Assume that there are two real numbers $r \geq \mathrm{e}, L>0$ such that

$$
|p(x)-p| \leq \frac{L}{\ln |x|} \quad \text { for all }|x| \geq r .
$$

Then $p(.) \in \mathcal{N}$ and so, $\mathfrak{M}$ is bounded on $L^{p(.)}\left(\mathbb{R}^{n}\right)$ by Theorem 6.5. 
(ii) Assume that we have for some $0<\alpha<1$

$$
|p(x)-p|=\frac{1}{\ln ^{\alpha}|x|} \quad \text { for all }|x| \geq \mathrm{e} .
$$

Then $p(.) \notin \mathcal{N}$.

Definition 6.7. Let $p(.) \in \mathfrak{B}\left(\mathbb{R}^{n}\right)$. Say that $p(.) \in \mathcal{A}$ if there exists a real number $p_{\infty}>1$ such that

$$
\left|\left\{x \in \mathbb{R}^{n} ;\left|p(x)-p_{\infty}\right| \geq \delta\right\} \cap B_{r}^{c}\right| \rightarrow 0 \text { provided } r \rightarrow \infty
$$

for each $\delta>0$.

Lemma 6.8. The inclusion $\mathcal{N} \subset \mathcal{A}$ holds.

Proof. See Lemma 3.2 from [21].

Lemma 6.9. Let $\mathfrak{p} \in \mathcal{B}, 1<p_{*} \leq p_{n} \leq p^{*}<\infty$ and $M: \ell^{\mathfrak{p}} \rightarrow \ell^{\mathfrak{p}}$ be bounded. Then the implication

$$
\sum_{n \in \mathbb{Z}} a_{n}^{\max \left\{p_{n}, p_{n+1}\right\}} \leq 1 \Rightarrow \sum_{n \in \mathbb{Z}} a_{n}^{\min \left\{p_{n}, p_{n+1}\right\}}<\infty
$$

holds holds for all $\mathfrak{a} \in \mathcal{M}_{+}$.

Proof. Set $\mathbb{Z}_{1}=\left\{n \in \mathbb{Z} ; p_{n}>p_{n+1}\right\}$ and $\mathbb{Z}_{2}=\left\{n \in \mathbb{Z} ; p_{n} \leq p_{n+1}\right\}$. Let $\mathfrak{a} \in \mathcal{M}_{+}$. Then

$$
\sum_{n \in \mathbb{Z}} a_{n}^{\max \left\{p_{n}, p_{n+1}\right\}}=\sum_{n \in \mathbb{Z}_{1}} a_{n}^{p_{n}}+\sum_{n \in \mathbb{Z}_{2}} a_{n}^{p_{n+1}} .
$$

Define sequences $\mathfrak{b}, \mathfrak{c} \in \mathcal{M}_{+}$by

$$
b_{n}=\left\{\begin{array}{ll}
a_{n} & \text { if } n \in \mathbb{Z}_{1}, \\
0 & \text { if } n \in \mathbb{Z}_{2} ;
\end{array} \quad c_{n}= \begin{cases}a_{n} & \text { if } n \in \mathbb{Z}_{2}, \\
0 & \text { if } n \in \mathbb{Z}_{1} .\end{cases}\right.
$$

Thus, $\sum_{n \in \mathbb{Z}} b_{n}^{p_{n}} \leq 1$ and $\sum_{n \in \mathbb{Z}} c_{n}^{p_{n}} \leq 1$. Since $M$ is bounded on $\ell^{\mathfrak{p}}$ we obtain

$$
\begin{aligned}
\infty & >\sum_{n \in \mathbb{Z}}(M \mathfrak{b})_{n}^{p_{n}} \\
& \geq \sum_{n \in \mathbb{Z}}\left(\frac{b_{n-1}+b_{n}}{2}\right)^{p_{n}} \\
& \geq 2^{-p^{*}} \sum_{n \in \mathbb{Z}} b_{n-1}^{p_{n}} \\
& =2^{-p^{*}} \sum_{n \in \mathbb{Z}} b_{n}^{p_{n+1}} \\
& =2^{-p^{*}} \sum_{n \in \mathbb{Z}_{1}} a_{n}^{p_{n+1}} .
\end{aligned}
$$


Analogously,

$$
\begin{aligned}
\infty & >\sum_{n \in \mathbb{Z}}(M \mathfrak{c})_{n}^{p_{n}} \\
& \geq \sum_{n \in \mathbb{Z}}\left(\frac{c_{n}+c_{n+1}}{2}\right)^{p_{n}} \\
& \geq 2^{-p^{*}} \sum_{n \in \mathbb{Z}} c_{n}^{p_{n}} \\
& =2^{-p^{*}} \sum_{n \in \mathbb{Z}_{2}} a_{n}^{p_{n}} .
\end{aligned}
$$

Thus, $\sum_{n \in \mathbb{Z}_{1}} a_{n}^{p_{n+1}}<\infty, \sum_{n \in \mathbb{Z}_{2}} a_{n}^{p_{n}}<\infty$ which finishes the proof.

Lemma 6.10. Let $1<p_{*} \leq p_{n} \leq p^{*}<\infty$ and $M: \ell^{\mathfrak{p}} \rightarrow \ell^{\mathfrak{p}}$ be bounded. Let $r, s$ be non-negative integers, $r \leq s$. Set $\mathbb{Z}_{n}(r, s)=\{k \in \mathbb{Z} ; n-r \leq k \leq$ $n+s\}$ for $n \in \mathbb{Z}$. Then the implication

$$
\sum_{n \in \mathbb{Z}} a_{n}^{\max } \mathbb{Z}_{n}(r, s) \leq 1 \Rightarrow \sum_{n \in \mathbb{Z}} a_{n}^{\min } \mathbb{Z}_{n}(r, s)<\infty
$$

holds.

Proof. The assertion is trivial for $r=s=0$. Let us prove the lemma for $r=0$ and $s>0$. Use the mathematical induction. For $s=1$ our implication follows from Lemma 6.9. Assume that the assertion is true for $s>1$. Let

$$
\sum_{n \in \mathbb{Z}} a_{n}^{\max } \mathbb{Z}_{n}(0, s+1) \leq 1 .
$$

Set $\mathbb{Z}_{1}=\left\{n \in \mathbb{Z} ; \min \mathbb{Z}_{n}(0, s+1)=\min \mathbb{Z}_{n}(0, s)\right\}, \mathbb{Z}_{2}=\{n \in$ $\left.\mathbb{Z} ; \min \mathbb{Z}_{n}(0, s+1)>p_{n+s+1}\right\}$. Clearly,

$$
\sum_{n \in \mathbb{Z}} a_{n}^{\min } \mathbb{Z}_{n}(0, s+1)=\sum_{n \in \mathbb{Z}_{1}} a_{n}^{\min \mathbb{Z}_{n}(0, s)}+\sum_{n \in \mathbb{Z}_{2}} a_{n}^{p_{n+s+1}}:=I_{1}+I_{2} .
$$

Let us estimate $I_{1}$. Set

$$
b_{n}= \begin{cases}a_{n} & \text { if } n \in \mathbb{Z}_{1}, \\ 0 & \text { if } n \notin \mathbb{Z}_{1} .\end{cases}
$$

Then

$$
\sum_{n \in \mathbb{Z}} b_{n}^{\max \mathbb{Z}_{n}(0, s)}=\sum_{n \in \mathbb{Z}_{1}} a_{n}^{\max \mathbb{Z}_{n}(0, s)} \leq 1
$$

and by the induction assumption we have

$$
I_{1}=\sum_{n \in \mathbb{Z}_{1}} a_{n}^{\min \mathbb{Z}_{n}(0, s)}=\sum_{n \in \mathbb{Z}} b_{n}^{\min \mathbb{Z}_{n}(0, s)}<\infty
$$


Estimate $I_{2}$. Set

$$
b_{n}= \begin{cases}a_{n} & \text { if } n \in \mathbb{Z}_{2}, \\ 0 & \text { if } n \notin \mathbb{Z}_{2} .\end{cases}
$$

Since $\max \mathbb{Z}_{n}(0, s+1)=\max \mathbb{Z}_{n}(0, s)$ for $n \in \mathbb{Z}_{2}$ we have by $(6.2)$

$$
\sum_{n \in \mathbb{Z}} b_{n}^{\max \mathbb{Z}_{n}(0, s)}=\sum_{n \in \mathbb{Z}_{2}} a_{n}^{\max \mathbb{Z}_{n}(0, s)}=\sum_{n \in \mathbb{Z}_{2}} a_{n}^{\max \mathbb{Z}_{n}(0, s+1)} \leq 1
$$

and by the induction assumption we obtain

$$
\sum_{n \in \mathbb{Z}} b_{n}^{p_{n}} \leq \sum_{n \in \mathbb{Z}} b_{n}^{\max \mathbb{Z}_{n}(0, s)}=\sum_{n \in \mathbb{Z}_{2}} a_{n}^{\max \mathbb{Z}_{n}(0, s+1)}:=D<\infty .
$$

Thus,

$$
\begin{aligned}
\sum_{n \in \mathbb{Z}}\left(\frac{b_{n}}{\max \{1, D\}^{1 / p_{*}}}\right)^{p_{n}} & \leq \sum_{n \in \mathbb{Z}}\left(\frac{b_{n}}{\max \{1, D\}^{1 / p_{n}}}\right)^{p_{n}} \\
& =\frac{1}{\max \{1, D\}} \sum_{n \in \mathbb{Z}} b_{n}^{p_{n}} \leq 1 .
\end{aligned}
$$

It shows that a sequence $\mathfrak{c}$ given by $c_{n}=\max \{1, D\}^{-1 / p_{*}} b_{n}$ satisfies $\sum_{n \in \mathbb{Z}} c_{n}^{p_{n}} \leq 1$. Since $M$ is bounded on $\ell^{\mathfrak{p}}$ we obtain

$$
\begin{aligned}
\infty & >\sum_{n \in \mathbb{Z}}(M \mathfrak{c})_{n}^{p_{n}} \\
& \geq \sum_{n \in \mathbb{Z}}\left(\frac{c_{n-s-1}+c_{n-s}+\cdots+c_{n}}{s+2}\right)^{p_{n}} \\
& \geq(s+2)^{-p^{*}} \sum_{n \in \mathbb{Z}} c_{n-s-1}^{p_{n}}=(s+2)^{-p^{*}} \sum_{n \in \mathbb{Z}} c_{n}^{p_{n+s+1}} \\
& \geq(s+2)^{-p^{*}} \max \{1, D\}^{-\frac{p^{*}}{p_{*}}} \sum_{n \in \mathbb{Z}} b_{n}^{p_{n+s+1}} \\
& =(s+2)^{-p^{*}} \max \{1, D\}^{-\frac{p^{*}}{p_{*}}} \sum_{n \in \mathbb{Z}_{2}} a_{n}^{p_{n+s+1}} .
\end{aligned}
$$

Thus, $I_{2}<\infty$ which proves with (6.3) and (6.4) the assertion for $s+1$.

Now, using the substitution $c_{n}=a_{-n}$ we can easily prove the assertion for all $r$ which finishes the proof.

In what follows we fix a sequence $\mathfrak{p} \in \mathcal{B}, 1<p_{*} \leq p_{n} \leq p^{*}<\infty$, and a continuous function $p($.) defined on $\mathbb{R}$ which satisfy for each $n \in \mathbb{Z}$

$$
\begin{aligned}
& \min \left\{p_{n}, p_{n+1}\right\} \leq p(t) \leq \max \left\{p_{n}, p_{n+1}\right\} \text { for } n \leq t \leq n+1, \\
& p(n)=p_{n} .
\end{aligned}
$$

We will prove that $\mathfrak{M}$ is bounded on $L^{p(x)}(\mathbb{R})$ provided $M$ is bounded on $\ell^{\mathfrak{p}}$ and $p(.) \in \mathcal{L}$. 
In [21] (see Lemma 2.7) is proved the following lemma.

Lemma 6.11. Let $p(.) \in \mathcal{L}, 1<\inf _{t \in \mathbb{R}} p(t) \leq p(x) \leq \sup _{t \in \mathbb{R}} p(t)<\infty$. Assume that a function $f$ satisfies $f(t)=0$ or $|f(t)| \geq 1$ for all $t \in \mathbb{R}$ and $\int_{\mathbb{R}}|f(t)|^{p(t)} d t \leq 1$. Then

$$
\int_{\mathbb{R}}|\mathfrak{M} f(x)|^{p(x)} \mathrm{d} x<\infty
$$

Denote for $f \in L_{1, l o c}(\mathbb{R})$

$$
\mathfrak{M}_{1} f(x)=\sup _{x \in I,|I| \leq 3} \frac{1}{|I|} \int_{I}|f(t)| d t, \quad \mathfrak{M}_{2} f(x)=\sup _{x \in I,|I| \geq 3} \frac{1}{|I|} \int_{I}|f(t)| d t
$$

where $I$ denotes an interval.

Lemma 6.12. Let $M$ is bounded on $\ell^{\mathfrak{p}}$ and $|f(t)| \leq 1$ for $t \in \mathbb{R}$, $\int_{\mathbb{R}}|f(t)|^{p(t)} d t \leq 1$. Then

$$
\int_{\mathbb{R}}\left|\mathfrak{M}_{2} f(t)\right|^{p(t)} d t<\infty
$$

Proof. Let $\mathfrak{a}$ be a sequence given by $a_{n}=\int_{n}^{n+1}|f(t)| d t$. Clearly, $a_{n} \leq 1$ for all $n \in \mathbb{Z}$. Since $|f(t)| \leq 1$ we obtain by Hölder inequality

$$
\begin{aligned}
\sum_{n \in \mathbb{Z}} a_{n}^{\max \left\{p_{n}, p_{n+1}\right\}} & \leq \sum_{n \in \mathbb{Z}} \int_{n}^{n+1}|f(t)|^{\max \left\{p_{n}, p_{n+1}\right\}} d t \\
& \sum_{n \in \mathbb{Z}} \int_{n}^{n+1}|f(t)|^{p(t)} d t \\
& =\int_{\mathbb{R}}|f(t)|^{p(t)} d t \\
& \leq 1 .
\end{aligned}
$$

By Lemma 6.9 we have

$$
\sum_{n \in \mathbb{Z}} a_{n}^{p_{n}} \leq \sum_{n \in \mathbb{Z}} a_{n}^{\min \left\{p_{n}, p_{n+1}\right\}}<\infty
$$

and since $M$ is bounded on $\ell^{\mathfrak{p}}$ we obtain

$$
\sum_{n \in \mathbb{Z}}(M \mathfrak{a})_{n}^{p_{n}}<\infty .
$$

Fix $x \in \mathbb{R}$. Estimate $\mathfrak{M}_{2} f(x)$. Denote $[x]$ the integer part of $x$ as usually. By the definition of $\mathfrak{M}_{2}$ we can choose $r \leq x \leq s$ such that $s-r \geq 3$ and

$$
\mathfrak{M}_{2} f(x) \leq \frac{2}{s-r} \int_{r}^{s}|f(t)| d t .
$$


Then

$$
\begin{aligned}
\mathfrak{M}_{2} f(x) & \leq \frac{2}{[s]-[r]-1} \int_{[r]}^{[s]+1}|f(t)| d t \\
& =\frac{2}{[s]-[r]-1} \sum_{k=[r]}^{[s]} \int_{k}^{k+1}|f(t)| d t \\
& =\frac{2([s]-[r]+1)}{[s]-[r]-1} \frac{1}{[s]-[r]+1} \sum_{k=[r]}^{[s]} a_{k} \\
& \leq 6(M \mathfrak{a})_{[x]} .
\end{aligned}
$$

Since $|f(t)| \leq 1$ for each real $t$ and consequently, $\left|\mathfrak{M}_{2} f(t)\right| \leq 1$ for each real $t$, we have

$$
\begin{aligned}
\int_{n}^{n+1}\left|\mathfrak{M}_{2} f(t)\right|^{p(t)} d t & \leq \int_{n}^{n+1}\left|\mathfrak{M}_{2} f(t)\right|^{\min \left\{p_{n}, p_{n+1}\right\}} d t \\
& \leq 6^{p *} \int_{n}^{n+1}(M \mathfrak{a})_{n}^{\min \left\{p_{n}, p_{n+1}\right\}} d t \\
& =6^{p *}(M \mathfrak{a})_{n}^{\min \left\{p_{n}, p_{n+1}\right\}} .
\end{aligned}
$$

By (6.8) we obtain

$$
\sum_{n \in \mathbb{Z}}(M \mathfrak{a})_{n}^{\max \left\{p_{n}, p_{n+1}\right\}} \leq \sum_{n \in \mathbb{Z}}(M \mathfrak{a})_{n}^{p_{n}}<\infty
$$

and by (6.7) we have

$$
\sum_{n \in \mathbb{Z}}(M \mathfrak{a})_{n}^{\min }\left\{p_{n}, p_{n+1}\right\}<\infty
$$

which gives

$$
\begin{aligned}
\int_{\mathbb{R}}\left|\mathfrak{M}_{2} f(t)\right|^{p(t)} d t & =\sum_{n \in \mathbb{Z}} \int_{n}^{n+1}\left|\mathfrak{M}_{2} f(t)\right|^{p(t)} d t \\
& \leq 6^{p *} \sum_{n \in \mathbb{Z}}(M \mathfrak{a})_{n}^{\min \left\{p_{n}, p_{n+1}\right\}} \\
& <\infty
\end{aligned}
$$

and finishes the proof.

Lemma 6.13. Let $p(.) \in \mathcal{L}, d>0$. Then there exists a constant $K(d, p()$.$) depending only on d$ and $p($.$) such that the implication$

$$
\int_{\mathbb{R}}|f(t)|^{p(t)} d t \leq 1 \Rightarrow \int_{I}|\mathfrak{M} f(t)|^{p(t)} d t \leq K(d, p(.))
$$

holds for all intervals $I \subset \mathbb{R},|I| \leq d$. 
Proof. In [2] the following assertion is proved. If $p(.) \in \mathcal{P},|I|<\infty$ then there is a constant $C(p(), I$.$) such that the implication$

$$
\int_{\mathbb{R}}|f(t)|^{p(t)} d t \leq 1 \Rightarrow \int_{I}|\mathfrak{M} f(t)|^{p(t)} d t \leq C(p(.), I) .
$$

An easy calculation of $C(p(), I$.$) shows that C(p(), I$.$) depends in fact on$ the function $p($.$) and only on the measure of |I|$ which finishes the proof.

Denote $p_{m, I}=\inf _{x \in I} p(x)$ and $p_{M, I}=\sup _{x \in I} p(x)$. As an easy consequence of the previous lemma we have the following lemma.

Lemma 6.14. Let $p(.) \in \mathcal{L}, d>0,0 \leq \alpha \leq 1$ and $K(d, p()$.$) be taken$ from Lemma 6.13. Then for each interval $I,|I| \leq d$ the implication

$$
\int_{\mathbb{R}}|f(t)|^{p(t)} d t \leq \alpha \Rightarrow \int_{I}|\mathfrak{M} f(t)|^{p(t)} d t \leq K(d, p(.)) \alpha^{\frac{p_{m, I}}{p_{M, I}}}
$$

holds.

Proof. Take $0<\alpha \leq 1$. Set

$$
g(t)=\alpha^{-\frac{1}{p_{M, I}}} f(t) \chi_{I}(t)
$$

Clearly,

$$
\begin{aligned}
\int_{\mathbb{R}}|g(t)|^{p(t)} d t & =\int_{I}\left|\alpha^{-\frac{1}{p_{M, I}}} f(t)\right|^{p(t)} d t \\
& \leq \int_{I}\left|\alpha^{-\frac{1}{p(t)}} f(t)\right|^{p(t)} d t \\
& =\frac{1}{\alpha} \int_{\mathbb{R}}|f(t)|^{p(t)} d t \\
& \leq 1 .
\end{aligned}
$$

By Lemma 6.13 we have

$$
\begin{aligned}
\alpha^{-\frac{p_{m, I}}{p_{M, I}}} \int_{I}\left|\mathfrak{M}\left(f \chi_{I}\right)(t)\right|^{p(t)} d t & \leq \int_{I} \alpha^{-\frac{p(t)}{p_{M, I}}}\left|\mathfrak{M}\left(f \chi_{I}\right)(t)\right|^{p(t)} d t \\
& =\int_{I}\left|\alpha^{-\frac{1}{p_{M, I}}} \mathfrak{M}\left(f \chi_{I}\right)(t)\right|^{p(t)} d t \\
& =\int_{I}|\mathfrak{M} g(t)|^{p(t)} d t \\
& \leq K(d, p)
\end{aligned}
$$

which finishes the proof.

Lemma 6.15. Let $p(.) \in \mathcal{L}$ and $M: \ell^{\mathfrak{p}} \rightarrow \ell^{\mathfrak{p}}$ be bounded and $\int_{\mathbb{R}}|f(t)|^{p(t)} d t \leq 1$. Then

$$
\int_{\mathbb{R}}\left|\mathfrak{M}_{1} f(t)\right|^{p(t)} d t<\infty
$$


Proof. Clearly, it suffices to prove the implication

$$
\int_{\mathbb{R}}|f(t)|^{p(t)} d t \leq \frac{1}{7} \Rightarrow \int_{\mathbb{R}}\left|\mathfrak{M}_{1} f(t)\right|^{p(t)} d t<\infty .
$$

Set $\alpha_{n}=\int_{n-3}^{n+4}|f(t)|^{p(t)} d t$. It is easy to see

$$
\begin{aligned}
\sum_{n \in \mathbb{Z}} \alpha_{n} & =\sum_{n \in \mathbb{Z}} \int_{n-3}^{n+4}|f(t)|^{p(t)} d t \\
& =7 \sum_{n \in \mathbb{Z}} \int_{n}^{n+1}|f(t)|^{p(t)} d t \\
& =7 \int_{\mathbb{R}}|f(t)|^{p(t)} d t \\
& \leq 1
\end{aligned}
$$

Then $\alpha_{n} \leq 1$ for each $n$ and by Lemma 6.14 we obtain

$$
\left.\int_{n-3}^{n+4} \mathfrak{M}\left(f \chi_{[n-3, n+4]}\right)(t)\right|^{p(t)} d t \leq K(7, p(.)) \alpha_{n}^{\frac{p_{m,[n-3, n+4]}}{p_{M,[n-3, n+4]}}} .
$$

Fix $n \in \mathbb{Z}$ and $t \in[n, n+1]$. Since $\mathfrak{M}_{1} f(t)$ depends only on values of $f(t)$ on the interval $[n-3, n+4]$ we have

$$
\int_{n}^{n+1}\left|\mathfrak{M}_{1} f(t)\right|^{p(t)} d t=\int_{n}^{n+1}\left|\mathfrak{M}_{1}\left(f \chi_{[n-3, n+4]}\right)(t)\right|^{p(t)} d t .
$$

Thus,

$$
\begin{aligned}
\int_{\mathbb{R}}\left|\mathfrak{M}_{1} f(t)\right|^{p(t)} d t & =\sum_{n \in \mathbb{Z}} \int_{n}^{n+1}\left|\mathfrak{M}_{1}\left(f \chi_{[n-3, n+4]}\right)(t)\right|^{p(t)} d t \\
& \leq \sum_{n \in \mathbb{Z}} \int_{n}^{n+1}\left|\mathfrak{M}\left(f \chi_{[n-3, n+4]}\right)(t)\right|^{p(t)} d t \\
& \leq \sum_{n \in \mathbb{Z}} K(7, p(.)) \alpha_{n}^{\frac{p_{m,[n-3, n+4]}}{p_{M,[n-3, n+4]}}} .
\end{aligned}
$$

Set $p_{m, n}=\min \left\{p_{n-3}, p_{n-2}, \ldots, p_{n+4}\right\}, p_{M, n}=\max \left\{p_{n-3}, p_{n-2}, \ldots, p_{n+4}\right\}$. Clearly, by (6.5) $p_{m,[n-3, n+4]}=p_{m, n}$ and $p_{M,[n-3, n+4]}=p_{M, n}$. Define $\beta_{n}$ by $\alpha_{n}=\beta_{n}^{p_{M, n}}$. By (6.9) we have $\sum_{n \in \mathbb{Z}} \beta_{n}^{p_{M, n}} \leq 1$ and by Lemma 6.10 we obtain $\sum_{n \in \mathbb{Z}} \beta_{n}^{p_{m, n}}<\infty$ which gives with (6.11)

$$
\int_{\mathbb{R}}\left|\mathfrak{M}_{1} f(t)\right|^{p(t)} d t \leq \sum_{n \in \mathbb{N}} K(7, p(.)) \alpha_{n}^{\frac{p_{m, n}}{p_{M, n}}}=K(7, p(.)) \sum_{n \in \mathbb{N}} \beta_{n}^{p_{m, n}}<\infty
$$

and finishes the proof. 
Theorem 6.16. Let $p(.) \in \mathcal{L}$ and assume that $M: \ell^{\mathfrak{p}} \rightarrow \ell^{\mathfrak{p}}$ is bounded. Then $\mathfrak{M}: L^{p(.)}(\mathbb{R}) \rightarrow L^{p(.)}(\mathbb{R})$ is bounded, too.

Proof. It suffices to prove the implication

$$
\int_{\mathbb{R}}|f(t)|^{p(t)} d t \leq 1 \Rightarrow \int_{\mathbb{R}} \mathfrak{M}|f(t)|^{p(t)} d t<\infty .
$$

Assume $\int_{\mathbb{R}}|f(t)|^{p(t)} d t \leq 1$. Write $f(t)=f \chi_{\{t ; f(t) \mid>1\}}(t)+$ $f \chi_{\{t ;|f(t)| \leq 1\}}(t):=f_{1}(t)+f_{2}(t)$. Clearly, $\mathfrak{M} f(t) \leq \mathfrak{M} f_{1}(t)+\mathfrak{M} f_{2}(t)$ and so,

$$
\begin{aligned}
\int_{\mathbb{R}}|\mathfrak{M} f(t)|^{p(t)} d t & \leq 2^{p^{*}}\left(\int_{\mathbb{R}}\left|\mathfrak{M}_{1} f(t)\right|^{p(t)} d t+\int_{\mathbb{R}}\left|\mathfrak{M}_{2} f(t)\right|^{p(t)} d t\right) \\
& =2^{p^{*}}\left(I_{1}+I_{2}\right) .
\end{aligned}
$$

By Lemma 6.11 we have $I_{1}<\infty$. Let us estimate $I_{2}$. Since $\mathfrak{M} f_{2}(t) \leq$ $\max \left\{\mathfrak{M}_{1} f_{2}(t), \mathfrak{M}_{2} f_{2}(t)\right\}$ it suffices to estimate

$$
I_{21}:=\int_{\mathbb{R}}\left|\mathfrak{M}_{1} f_{2}(t)\right|^{p(t)} d t, \quad I_{22}:=\int_{\mathbb{R}}\left|\mathfrak{M}_{2} f_{2}(t)\right|^{p(t)} d t
$$

But $I_{21}<\infty$ by Lemma 6.15 and $I_{22}<\infty$ by Lemma 6.12 which finishes the proof.

Theorem 6.17. There is a function $p(.) \in \mathcal{L} \cap(\mathcal{A} \backslash \mathcal{N})$ such that $\mathfrak{M}$ is bounded on $L^{p(.)}(\mathbb{R})$.

Proof. Choose real numbers $\alpha, L$ with $1<\alpha \leq 2, L>1$. Set $K_{n}=\left[L^{n^{\alpha}}\right]$ and $r_{n}=\frac{1}{n}$ for $n \in \mathbb{N}, r_{0}=1$. Let the sequence $\mathfrak{p}$ be given by (2.3) and the function $p$ be given by

$$
p(t)=\sum_{n \in \mathbb{Z}}\left(p_{n+1}(t-n)+p_{n}(n+1-t)\right) \chi_{[n, n+1]}(t) .
$$

Since $p($.$) is bounded and linear on each interval [n, n+1]$ the function $p($.$) is Lipschitz and consequently, p(.) \in \mathcal{L}$. Evidently, $p($.$) satisfies (6.5).$ The operator $M$ is bounded on $\ell^{\mathfrak{p}}$ by Lemma 5.8 and so, $\mathfrak{M}$ is bounded on $L^{p(.)}(\mathbb{R})$ by Theorem 6.16. It remains to prove $p(.) \in \mathcal{L} \cap(\mathcal{A} \backslash \mathcal{N})$. Clearly, $p(.) \in \mathcal{L}$ by the assumption. Moreover, by Definition 2.16 $\lim _{|n| \rightarrow \infty} p_{n}=p+\lim _{|n| \rightarrow \infty} r_{n}=p+\lim _{|n| \rightarrow \infty} \frac{1}{n}=p$ and thus, $p(.) \in \mathcal{A}$. Let us prove $p(.) \notin \mathcal{N}$. Choose an arbitrary $c, 0<c<1$. Estimate

$$
\begin{aligned}
\int_{\mathbb{R}}|p(t)-p| c^{\frac{1}{p(t)-p \mid}} d t & \geq \sum_{n=1}^{\infty} \int_{1+A_{n-1}}^{A_{n}} r_{n} c^{\frac{1}{r_{n}}} d t=\sum_{n=1}^{\infty}\left(K_{n}-1\right) \frac{c^{n}}{n} \\
& =\sum_{n=1}^{\infty}\left[L^{n^{\alpha}}\right] \frac{c^{n}}{n}-\sum_{n=1}^{\infty} \frac{c^{n}}{n} \geq \sum_{n=1}^{\infty} \frac{L^{n^{\alpha}} c^{n}}{n}-2 \sum_{n=1}^{\infty} \frac{c^{n}}{n}
\end{aligned}
$$


Since $c<1$ we have $\sum_{n=1}^{\infty} \frac{c^{n}}{n}<\infty$. Since $L>1$ and $\alpha>1$ we obtain $\sum_{n=1}^{\infty} \frac{L^{n^{\alpha}} c^{n}}{n}=\infty$ which gives $p(.) \notin \mathcal{N}$ and finishes the proof.

It would be undoubtedly interesting to estimate the asymptotic behavior of $p($.$) at infinity given by (6.12) and compare it with Example 6.6.$

Lemma 6.18. Let $\alpha>1, L>1, K_{n}=\left[L^{n^{\alpha}}\right], r_{n}=\frac{1}{n}$ and $p($.$) be$ given by (6.12). Then there are two numbers $c_{1}, c_{2}>0$ such that inequalities

$$
\frac{c_{1}}{(\ln |x|)^{1 / \alpha}} \leq|p(x)-p| \leq \frac{c_{2}}{(\ln |x|)^{1 / \alpha}}
$$

hold for $|x| \geq \mathrm{e}$.

Proof. It suffices to find $c>1, c_{1}, c_{2}>0$ such that

$$
\frac{c_{1}}{(\ln x)^{1 / \alpha}} \leq p(x)-p \leq \frac{c_{2}}{(\ln x)^{1 / \alpha}} \quad \text { for all } \quad x>c .
$$

Take $m \in \mathbb{N}$ such that for each $n \in \mathbb{N}, n>m$ the following inequalities hold:

$$
\begin{aligned}
& \ln n \leq \alpha(\ln L) n^{\alpha-1} \\
& L^{n^{\alpha}} \leq 2\left[L^{n^{\alpha}}\right] ; \\
& 2 \ln 2 \leq(\ln L) n^{\alpha} \quad \text { and } \quad \ln 2 \leq(\ln L)(n+1)^{\alpha} .
\end{aligned}
$$

Fix $n>m$. By (2.2) and (6.15) we have

$$
L^{n^{\alpha}} \leq 2\left[L^{n^{\alpha}}\right]=2 K_{n} \leq 2 \sum_{j=1}^{n} K_{j}=2 A_{n} .
$$

By the Lagrange mean value theorem we obtain

$$
\alpha n^{\alpha-1} \leq(n+1)^{\alpha}-n^{\alpha} \leq \alpha(n+1)^{\alpha-1}
$$

which gives with (6.14)

$$
\ln n \leq \alpha(\ln L) n^{\alpha-1} \leq(\ln L)\left((n+1)^{\alpha}-n^{\alpha}\right)
$$

and so, $n L^{n^{\alpha}} \leq L^{(n+1)^{\alpha}}$. As a consequence we obtain

$$
\begin{aligned}
& A_{n+1}=K_{1}+K_{2}+\cdots+K_{n}+K_{n+1} \leq n K_{n}+K_{n+1} \\
& \leq n L^{n^{\alpha}}+L^{(n+1)^{\alpha}} \leq 2 L^{(n+1)^{\alpha}} .
\end{aligned}
$$

Choose $x \in\left[A_{n}, A_{n+1}\right]$. By (2.3) and (6.12) we have

$$
\frac{1}{n+1} \leq p(x)-p \leq \frac{1}{n}
$$

Moreover, due to (6.17) and (6.18) we obtain

$$
\frac{1}{2} L^{n^{\alpha}} \leq x \leq 2 L^{(n+1)^{\alpha}} .
$$


These inequalities can be rewritten as

$$
n^{\alpha} \ln L-\ln 2 \leq \ln x \leq(n+1)^{\alpha} \ln L+\ln 2
$$

Consequently, by (6.16) we have

$$
\frac{1}{2} n^{\alpha} \ln L \leq \ln x \leq 2(n+1)^{\alpha} \ln L
$$

which yields

$$
\left(\frac{1}{2 \ln L}\right)^{1 / \alpha} \frac{1}{n+1} \leq \frac{1}{(\ln x)^{1 / \alpha}} \leq\left(\frac{2}{\ln L}\right)^{1 / \alpha} \frac{1}{n} .
$$

Since $\frac{1}{n} \leq \frac{2}{n+1}$ we have by $(6.19)$

$$
\begin{aligned}
& \frac{1}{2}\left(\frac{1}{2 \ln L}\right)^{1 / \alpha}(p(x)-p) \leq \frac{1}{2}\left(\frac{1}{2 \ln L}\right)^{1 / \alpha} \frac{1}{n} \leq \frac{1}{(\ln x)^{1 / \alpha}} \\
& \leq 2\left(\frac{2}{\ln L}\right)^{1 / \alpha} \frac{1}{n+1} \leq 2\left(\frac{2}{\ln L}\right)^{1 / \alpha}(p(x)-p) .
\end{aligned}
$$

We have proved for $n>m$ and $x \in\left[A_{n}, A_{n+1}\right]$

$$
\frac{\frac{1}{2}\left(\frac{\ln L}{2}\right)^{1 / \alpha}}{(\ln x)^{1 / \alpha}} \leq p(x)-p \leq \frac{2(2 \ln L)^{1 / \alpha}}{(\ln x)^{1 / \alpha}} .
$$

Now, the easy fact $\left[A_{n}, \infty\right]=\bigcup_{n>m}\left[A_{n}, A_{n+1}\right]$ proves (6.13) with $c=$ $A_{m+1}, c_{1}=\frac{1}{2}\left(\frac{\ln L}{2}\right)^{1 / \alpha}$ and $c_{2}=2(2 \ln L)^{1 / \alpha}$.

Remark 6.19. Let $\alpha>0, L>1, K_{n}=\left[L^{n^{\alpha}}\right], r_{n}=\frac{1}{n}$ and $p($.$) be$ given by (6.12).

If $0<\alpha \leq 1$ then we have $|p(x)-p| \leq \frac{c}{\ln |x|}$ which gives $p(.) \in \mathcal{N}$ by Example $6.6(i)$ and so, $\mathfrak{M}$ is bounded on $L^{p(\cdot)}(\mathbb{R})$ again by Example 6.6 (i).

If $1<\alpha \leq 2$ then the function $p()-$.$p decreases to 0$ comparably with $\frac{1}{\ln |x|^{1 / \alpha}}$ and so, $p(.) \notin \mathcal{N}$ by Example 6.6 (ii) but $\mathfrak{M}$ is bounded on $L^{p(.)}(\mathbb{R})$ by Lemma 6.17.

\section{References}

[1] D. Cruz-Uribe, A. Fiorenza and C. J. Neugebauer, The maximal function on variable $L^{p}$ spaces, Ann. Acad. Sci. Fenn. Math., 28 (1) (2003), 223238.

[2] L. Diening, Maximal function on generalized Lebesgue spaces $L^{p(.)}$, Math. Inequal. Appl., 7 (2) (2004), 245-253.

[3] L. Diening and M. Růžička, Calderón-Zigmund operators on generalied Lebesgue spaces $L^{p(.)}$ and problems related to fluid dynamics, J. Reine Angew. Math., 563 (2003), 197-220. 
[4] L. Diening and M. Růžička, Riesz potencial and Sobolev embeddings on generalized Lebesgue and Sobolev spaces $L^{p(.)}$ and $W^{1, p(.)}$, Math. Nachr., 268 (2004), 31-43.

[5] L. Diening and M. Růžička, Integral operators on the halfspace in generalized Lebesque spaces $L^{p(.)}$. I, J. Math. Anal. Appl., 298 (2) (2004), 559-571.

[6] L. Diening and M. Růžička, Integral operators on the halfspace in generalized Lebesque spaces $L^{p(.)}$. II, J. Math. Anal. Appl., 298 (2) (2004), 572-588.

[7] D.E. Edmunds, J. Lang and A. Nekvinda, On $L^{p(x)}$ norms, Proc. R. Soc. Lond. A, 455 (1999), 219-225.

[8] D. E. Edmunds and A. Nekvinda, Averaging operators on $l^{\mathfrak{p}}$ and $L^{p(x)}$, Math. Inequal. Appl., 5 (2) (2002), 235-246.

[9] D.E. Edmunds and J. Rákosník, Sobolev embeddings with variable exponent, Studia Math., 143 (2000), 267-293.

[10] X. Fan, J. Shen and D. Zhao, Sobolev Embedding Theorems for Spaces $W^{k \cdot p(x)}(\Omega)$, J. Math. Anal. Appl., 262 (2001), 749-760.

[11] X. Fan, Y. Zhao and D. Zhao, Compact imbeddings theorems with symetry of Strass-Lions type for the space $W^{k, p(x)}(\Omega)$, J. Math. Anal. Appl., 255 (2001), 333-348.

[12] E. Hewitt and K. Stromberg, Real and Abstract Analysis, SpringerVerlag Berlin Heidelberg New York, 1965.

[13] V. Kokilashvili and S. Samko, Maximal and fractional operators in weighted $L^{p(x)}$ spaces, Rev. Mat. Iberoamericana, 20 (2) (2004), 493515.

[14] V. Kokilashvili and S. Samko, On Sobolev theorem for Riesz type potencials in Lebesque spaces with variable exponent, Z. Anal. Anwendungen, 22 (4) (2003), 899-910.

[15] V. Kokilashvili and S. Samko, Singular integrals and potentials in some Banach function spaces with variable exponent, J. Funct. Spaces Appl., 1 (1) (2003), 45-59.

[16] V. Kokilashvili and S. Samko, Singular intregrals in weighted Lebesgue spaces with variable exponent, Georgian Math. J., 10 (1) (2003), 145156.

[17] O. Kováčik and J. Rákosník, On spaces $L^{p(x)}$ and $W^{k, p(x)}$, Czech. Math. J., 41 (1996), 167-177. 
[18] F. J. Martín-Reyes, Weights, one-sided operators, singular integrals and ergodic theorem, in Proceedings of Nonlinear Analysis, Function Spaces and Applications, Prometheus, Prague, 5 (1994), 103-137.

[19] L. Pick and M. Růžička, An example of a space $L^{p(x)}$ on which the Hardy-Littlewood maximal operator is not bounded, Expositiones Mathematicae, 19 (2001), 369-371.

[20] A. Nekvinda, Equivalence of $\ell^{\left\{p_{n}\right\}}$ norms and shift operators, Math. Inequal. Appl., 5 (4) (2002), 711-723.

[21] A. Nekvinda, Hardy-Littlewood maximal operator on $L^{p(x)}\left(\mathbb{R}^{n}\right)$, Math. Inequal. Appl., 7 (2) (2004), 255-265.

[22] M. Růžička, Electrorheological fluids: Modeling and mathematical theory, Lecture Notes in Mathematics, 1748, Berlin: Springer, xvi, 2000.

[23] M. Růžička, Flow of shear dependent electrorheological fluids, C.R.Acad.Sci.Paris Série, I 329 (1999), 393-398.

Department of Mathematics

Faculty of Civil Engineering

Czech Technical University

Thákurova 7

16629 Prague 6

Czech Republic

(E-mail : nekvinda@fsv.cvut.cz)

(Received : December 2005) 


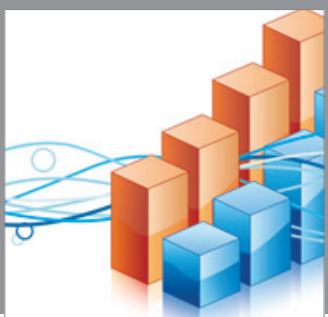

Advances in

Operations Research

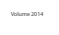

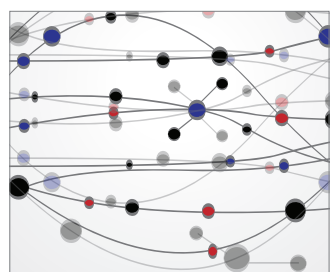

\section{The Scientific} World Journal
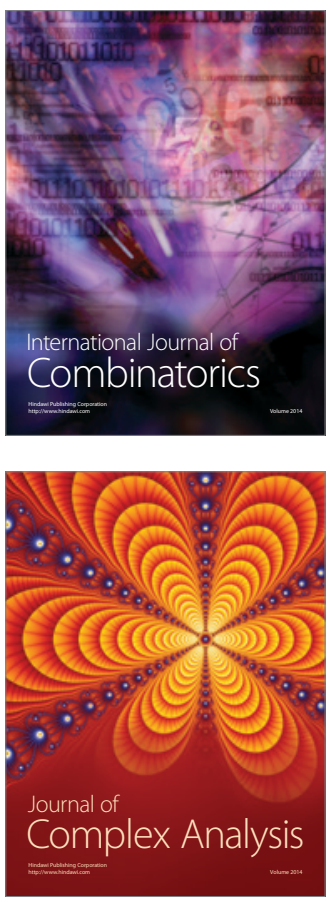

International Journal of

Mathematics and

Mathematical

Sciences
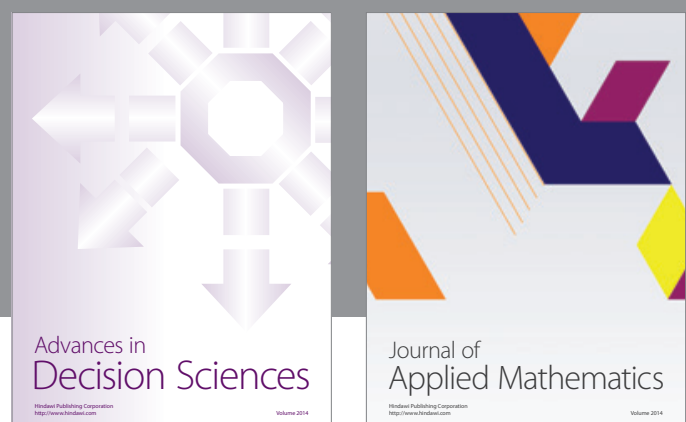

Journal of

Applied Mathematics
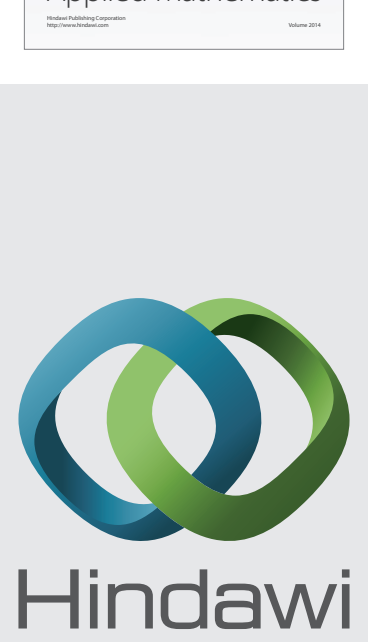

Submit your manuscripts at http://www.hindawi.com
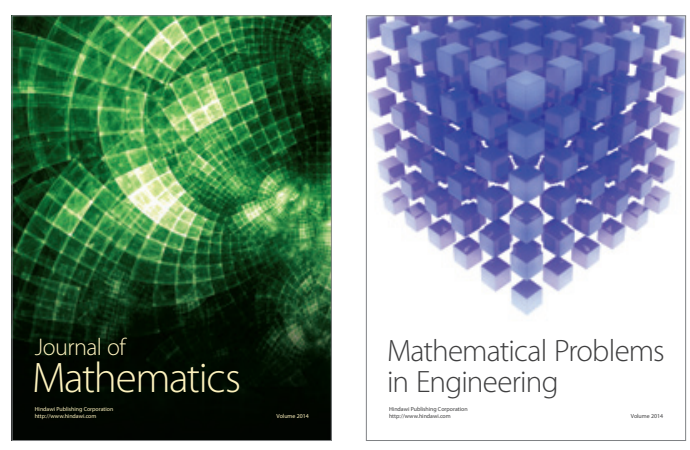

Mathematical Problems in Engineering
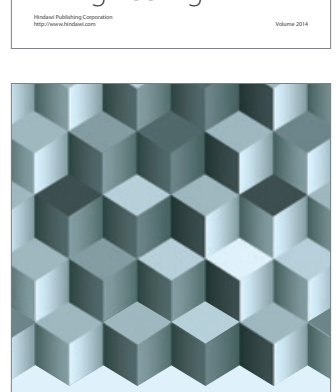

Journal of

Function Spaces
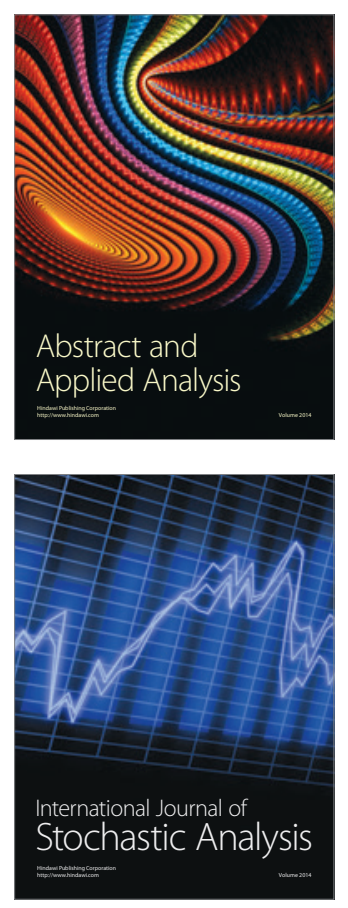

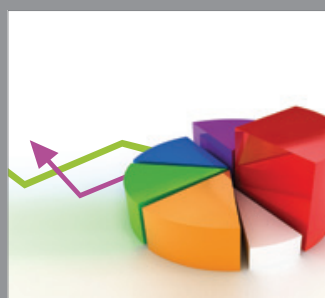

ournal of

Probability and Statistics

Promensencen
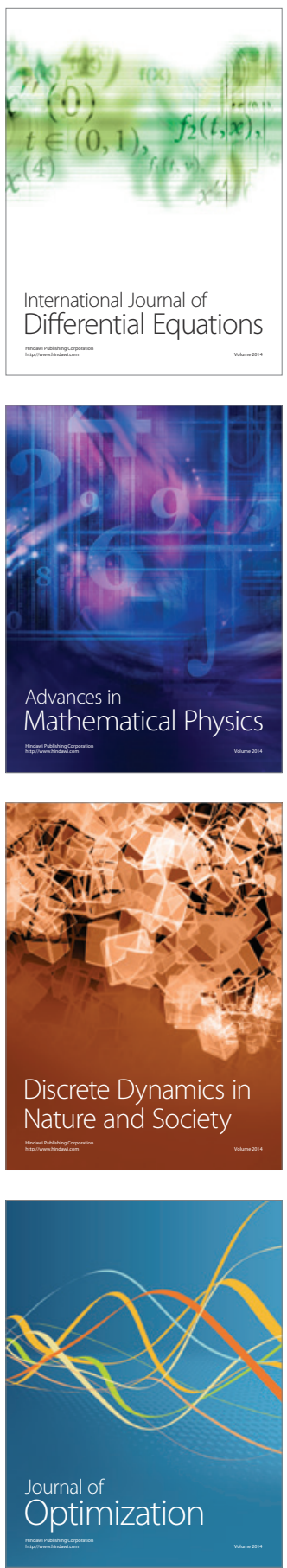